This item was submitted to Loughborough's Research Repository by the author.

Items in Figshare are protected by copyright, with all rights reserved, unless otherwise indicated.

\title{
Automatic clustering using a local search-based human mental search algorithm for image segmentation
}

PLEASE CITE THE PUBLISHED VERSION

https://doi.org/10.1016/j.asoc.2020.106604

PUBLISHER

Elsevier

VERSION

AM (Accepted Manuscript)

\section{PUBLISHER STATEMENT}

This paper was accepted for publication in the journal Applied Soft Computing and the definitive published version is available at https://doi.org/10.1016/j.asoc.2020.106604.

\section{LICENCE}

CC BY-NC-ND 4.0

\section{REPOSITORY RECORD}

Mousavirad, Seyed Jalaleddin, Hossein Ebrahimpour-Komleh, and Gerald Schaefer. 2020. "Automatic Clustering Using a Local Search-based Human Mental Search Algorithm for Image Segmentation". Loughborough University. https://hdl.handle.net/2134/14236634.v1. 


\title{
Automatic Clustering Using a Local Search-Based Human Mental Search Algorithm for Image Segmentation
}

\author{
Seyed Jalaleddin Mousavirad ${ }^{1}$, Hossein Ebrahimpour-Komleh ${ }^{2}$, and Gerald Schaefer ${ }^{3}$ \\ ${ }^{1}$ Faculty of Engineering, Sabzevar University of New Technology, Sabzevar, Iran \\ ${ }^{2}$ Department of Electrical and Computer Engineering, University of Kashan, Iran \\ ${ }^{3}$ Department of Computer Science, Loughborough University, U.K.
}

\begin{abstract}
Clustering is a commonly employed approach to image segmentation. To overcome the problems of conventional algorithms such as getting trapped in local optima, in this paper, we propose an improved automatic clustering algorithm for image segmentation based on the human mental search (HMS) algorithm, a recently proposed method to solve complex optimisation problems. In contrast to most existing methods for image clustering, our approach does not require any prior knowledge about the number of clusters but rather determines the optimal number of clusters automatically. In addition, for further improved efficacy, we incorporate local search operators which are designed to make changes to the current cluster configuration.

To evaluate the performance of our proposed algorithm, we perform an extensive comparison with several state-ofthe-art algorithms on a benchmark set of images and using a variety of metrics including cost function, correctness of the obtained numbers of clusters, stability, as well as supervised and unsupervised segmentation criteria. The obtained results clearly indicate excellent performance compared to existing methods with our approach yielding the best result in 16 of 17 cases based on cost function evaluation, 9 of 11 cases based on number of identified clusters, 13 of 17 cases based on the unsupervised Borsotti image segmentation criterion, and 7 of 11 cases based on the supervised PRI image segmentation metric.
\end{abstract}

Keywords: Image segmentation, automatic clustering, human mental search, metaheuristic.

\section{Introduction}

Image segmentation plays a fundamental role in image analysis while posing a challenging task since so far no image segmentation algorithm has been proposed that is capable of handling a variety of images with satisfactory quality. Image segmentation is one of the most complex tasks in computer vision [1], and is a crucial pre-processing step for a variety of applications [2-5]. The purpose of image segmentation is to divide an image into non-overlapping areas so that pixels located in the same area have similar characteristics.

One of the most commonly employed methods for image segmentation is clustering. Clustering is applied to identify 
similar groups (clusters) in an image so that the samples associated with a group share similar features, while the samples belonging to different groups have different features. Clustering in images is challenging in comparison to clustering other types of data [6] because the number of samples (pixels) in an image is high since even a relatively small image of size $256 \times 256$ has 65,536 pixels, while an $512 \times 512$ image has 262,144 samples.

The $k$-means algorithm [7] is one of the most popular clustering algorithms. Here, $k$ refers to the number of clusters, which needs to be specified in advance, while the algorithm minimises a cost function that is defined by the distances of the samples to their cluster centres. Fuzzy $c$-means (FCM) [8] is a soft version of $k$-means, where a pattern belongs to multiple clusters, whereas $k$-means assigns a pattern to exactly one cluster. Even though these and similar conventional algorithms are widely adopted, they have some inherent disadvantages including sensitivity to the initial cluster centres, getting trapped in local optima, and the need to specify the number of clusters a priori.

Metaheuristic algorithms [9] such as differential evolution (DE) [10], particle swarm optimisation (PSO) [11], and harmony search (HS) [12] are a way to overcome the problem of getting stuck in local optima. Metaheuristic algorithms are a type of problem-independent algorithm that use stochastic operators to solve an optimisation problem. These algorithms have attracted a lot of attention not only because, compared to traditional optimisation algorithms, they are significantly better in escaping from local optima, but also as they do not require calculation of the gradient of the cost function. Metaheuristic algorithms can be divided into two categories: single solution-based and population-based approaches. Single-based metaheuristics start from a single random candidate solution, which they then, in an iterative process, try to improve. In contrast, population-based algorithms start from a set of candidate solutions. Information can be shared among the candidate solutions, while the main advantage of population-based methods is their greater ability to avoid getting trapped in a local optimum. Two key parts in the design of population-based metaheuristics are intensification and diversification. Intensification refers to trying to find better solutions around a good candidate solution, while diversification is the ability to effectively explore the search space to identify good diverse solutions. These two criteria are usually in conflict with each other so that finding a balance between them is one of the critical challenges in developing high-performing metaheuristic algorithms.

In recent years, there has been much interest in using population-based metaheuristic algorithms for clustering-based image segmentation. A genetic algorithm (GA) [13] 15] was one of the first such algorithms employed to image clustering, with [16] introducing a hybrid method combining $k$-means with a GA and showing that this combination is less sensitive to initialisation. [17] presented a method based on a combination of FCM and GA to segment satellite images using a cost function based on the total weighted squared error. Particle swarm optimisation (PSO) [11] is another populationbased metaheuristic algorithm that has been frequently utilised for image segmentation. [18] proposed finding the cluster centres using PSO, with the structure of each particle being a one-dimensional array equal to the number of clusters. They compared their algorithm with ISODATA, showing the PSO approach to be more effective. In [19], a chaotic PSO algorithm for image segmentation was introduced, where the chaotic part was designed to reduce the probability of getting trapped in local optima leading to better performance compared to a standard PSO. In [20], a barebones PSO (BPSO) algorithm for image segmentation was proposed, where the PSO parameter values were obtained automatically, leading to improved efficacy compared to PSO. Recently, [21] proposed an image clustering method based on an improved PSO that adapts the inertia weight and learning parameters dynamically and according to the fitness value. Results on 
synthetic and MRI images showed this to give better results compared to some conventional and PSO algorithms. Other state-of-the-art metaheuristic algorithms that have been used for clustering-based image segmentation include differential evolution [22], the artificial bee algorithm [23], and harmony search [24].

In the clustering-based image segmentation methods above, the metaheuristic algorithms were used to try to find optimal cluster centres, while the number of clusters needed to be specified in advance. In practical applications, it is more likely that the number of clusters is unknown a priori or even that estimating it approximately is not possible [25]. For example, an autonomous robot may need to detect obstacles and goals in its vicinity using an in-built digital camera. In such a case, for successful operation, the robot needs to perform automatic clustering on the captured images to detect any number of objects since prior knowledge on this will not be available. Consequently, finding the optimal number of clusters, or automatic clustering, in an image has become an important research topic [26]. Although there are several studies on automatic image clustering, their results are still not satisfactory and identifying the optimal number of clusters remains a challenging task. Furthermore, the No Free Lunch theorem [27] explains that there is no best algorithm to solve all optimisation problems, and thus motivates further research on new algorithms and their application to new problem domains.

To the best of our knowledge, only a handful of metaheuristic algorithms have been employed for automatic image clustering. [28] proposed a method based on PSO for automatic clustering-based image segmentation, using a binary PSO to find the optimal number of clusters. The selected cluster centres were then modified by $k$-means, after which again the binary PSO algorithm was employed, with this iterative process continuing until a convergence criterion was met. They used synthetic and real images to evaluate the efficacy of their method and showed it to perform well. In [25], a DE algorithm was used for automatic clustering. They used an array of length $K_{\max }+K_{\max } \times d$, where the first $K_{\max }$ elements are floating-point numbers in the interval $[0,1]$ that specify active and inactive clusters, while the remaining elements specify the cluster centres. In their results, competitive efficacy compared to PSO and GA was shown. [1] introduced an improved algorithm for automatic fuzzy image clustering. The encoding strategy here was similarly an array of length $K_{\max }+K_{\max } \times d$, while the employed cost function was based on a cluster validity index. Results on multiple images and compared with two other algorithms, automatic fuzzy clustering differential evolution and fuzzy variable string genetic algorithm, suggested superiority of the proposed algorithm. A harmony search algorithm for automatic image clustering was proposed in [29]. Here, a real-coded variable-length harmony vector was used together with a modified Euclidean distance to assign each sample to its corresponding cluster. Although there are not many studies on population-based automatic image clustering yet, automatic clustering has also been investigated in other fields such as gene functional enrichment analysis [30].

In order to improve the efficacy of metaheuristic algorithms for clustering-based image segmentation, some researchers have employed local search mechanisms. The most popular and most widely used algorithm in this regard is $k$-means. [31] combined a colonial competition algorithm with $k$-means for static clustering problems, while [32] used DE in combination with $k$-means to obtain improved results compared to the original DE algorithm. $k$-means has also been combined with other population-based metaheuristic algorithms including the electromagnetism-like mechanism [33] and the gravitational search algorithm [34]. However, incorporating a local search mechanism into a metaheuristic has typically been employed for static rather than automatic clustering. 
The human mental search (HMS) algorithm [35] is a recent population-based metaheuristic algorithm inspired by the strategy of searching in the bid space of an online auction. The algorithm has three main stages: mental search, which searches around candidate solutions using a Levy flight mechanism; bid grouping, which finds promising areas using a clustering algorithm; and moving candidate solutions towards promising areas. HMS has been shown to provide competitive performance compared to other metaheuristic algorithms [6, 35, 36] due to its powerful operators. For example, the update in HMS algorithm is based on a region rather than a point which better allows HMS to prevent early convergence. In our earlier work, we have applied HMS to image clustering in [6] which has demonstrated the algorithm's ability to improve upon other image clustering approaches. There, our encoding representation was a one-dimensional array defining the cluster centres, while three different cost functions were evaluated. The obtained results showed HMS to be superior to other metaheuristic algorithms. However, an a priori specification of the number of clusters was required. In this paper, we remove this requirement by changing the encoding strategy and cost function, while we further improve the efficacy of the algorithm by incorporating a local search strategy.

The aim of this paper is to propose a novel approach to automatic image clustering based on the HMS algorithm. One of the main features of our ACLSHMS (Automatic Clustering Local Search HMS) algorithm is that it does not require any prior knowledge about the number of clusters as it is capable of simultaneously finding both the optimal number of clusters and their cluster centres. To further improve the performance of the proposed algorithm, a local search strategy for HMS is introduced that allows to change the cluster configuration. It comprises four operators, (1) changing cluster centres, (2) changing active clusters, (3) activating clusters, and (4) deactivating clusters, while during each iteration of the algorithm neighbouring solutions around existing candidate solutions are generated based on these operators. To evaluate the performance of our proposed algorithm, we perform an extensive comparison with several state-of-the-art algorithms on a benchmark set of images and using a variety of metrics including cost function, correctness of the obtained numbers of clusters, stability, as well as supervised and unsupervised segmentation criteria. The obtained results clearly indicate excellent performance of ACLSHMS compared to existing methods. The main contributions of our paper are summarised as follows:

- We propose an automatic image clustering for image segmentation based on the human mental search algorithm.

- The encoding strategy and objective function used allow to simultaneously identify not only the cluster centres but also the optimal number of clusters.

- We incorporate a local search strategy to improve improve the performance of our algorithm.

- Extensive experimental results demonstrate our proposed ACLSHMS algorithm to outperform several other populationbased metaheuristic algorithms on a variety of evaluation measures.

The remainder of the paper is organised as follows. In Section 2, we explain basic concepts of clustering and the HMS algorithm. In Section 3, we then detail our proposed algorithm. Section 4 provides extensive experimental results of the proposed method including a comprehensive comparison with other methods. Finally, Section 5 concludes the paper. 


\section{Background}

\subsection{Pattern clustering}

The purpose of clustering is to group patters into clusters, which is generally performed by minimising the distances of samples within a cluster (intra-cluster distances), while maximising the distances between samples from different clusters (inter-cluster distances). More formally, assume a set of $N$ patterns as $P=\left\{p_{1}, p_{2}, \ldots, p_{N}\right\}$ with $p_{i}=\left\{p_{i, 1}, p_{i, 2}, \ldots, p_{i, N}\right\}$. Clustering tries to find $K$ partitions $C=\left\{c_{1}, c_{2}, \ldots, c_{K}\right\}$ such that

1. $c_{i} \neq \emptyset, i=1 \ldots K$

2. $\bigcup_{i=1}^{K} c_{i}=\mathrm{P}$;

3. $c_{i} \cap c_{j}=\varphi, i, j=1 \ldots K, i \neq j$.

Generally speaking, samples are assigned to a cluster using a similarity criterion. In this paper, we use the Euclidean distance as the employed similarity criterion.

\subsection{Human mental search}

Human mental search (HMS) [35] is a recent population-based metaheuristic algorithm inspired by the exploration of the bid space in online auctions. Algorithm 1 summarises the HMS algorithm in the form of pseudo-code, while in the following the operators of HMS algorithm are explained in more detail.

\subsubsection{Initialisation}

Since the algorithm is population-based, an initial population of candidate solutions needs to be created. In HMS, a candidate solution is called a bid, and candidate solutions are initialised randomly. The cost value of each bid in the initial population, which specifies the quality of a bid, is then calculated.

\subsubsection{Mental search}

The aim of the mental search operator is to produce a series of new bids around a bid in the population using a Levy flight, i.e., a random walk in which the step size follows the Levy distribution. A random walk is a type of Markov chain in which a position is solely dependent on its previous position, while one of the features of Levy flight is that it produces many small steps and a few big steps in a movement sequence. This allows the HMS algorithm to seek diversification and intensification simultaneously. A new bid in mental search is obtained by

$$
N S=b i d+S,
$$

with $S$ calculated as

$$
S=\left(2-N F E\left(2 / N F E_{\text {max }}\right)\right) \alpha \oplus L e v y,
$$




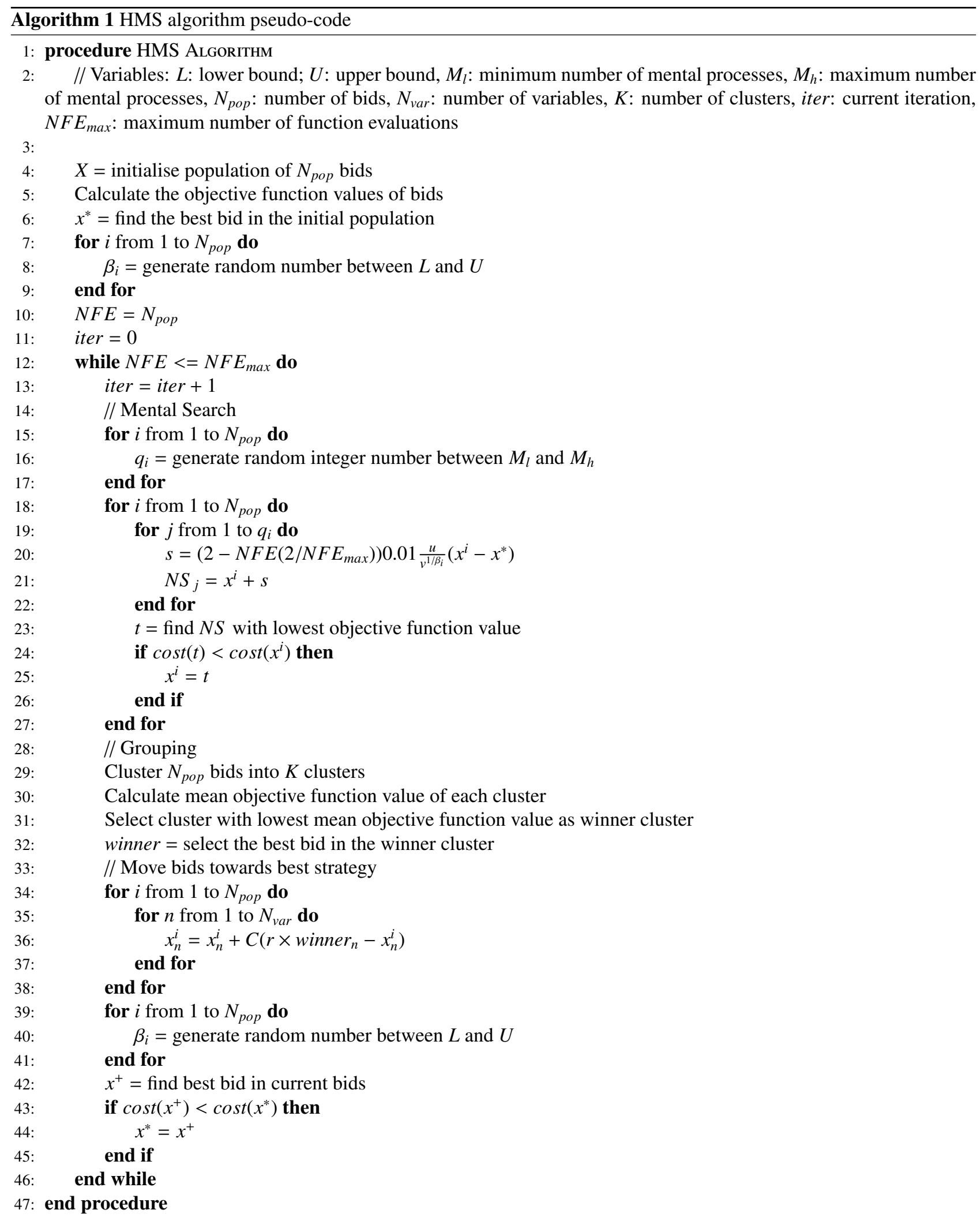

where $N F E_{\text {max }}$ is the maximum number of function evaluations, $N F E$ is the current number of function evaluations, $\alpha$ is a random number, and $\oplus$ signifies entry-wise multiplication. The coefficient $\left(2-N F E *\left(2 / N F E_{\text {max }}\right)\right)$ is called the descending factor which starts from a value 2 and tends towards 0 with an increasing number of iterations. At the beginning of the algorithm, larger values result in larger jumps leading to diversification, while later the algorithm turns from diversification to intensification with smaller jumps. 
Eq.2] is modified to [35]

$$
S=\left(2-N F E *\left(2 / N F E_{\text {max }}\right)\right) * 0.01 * \frac{u}{v^{1 / \beta}} *\left(x^{i}-x^{*}\right),
$$

where $x^{*}$ is the best candidate solution found so far, and $u$ and $v$ are two random numbers generated from a normal distribution as

$$
u \sim N\left(0, \sigma_{u}^{2}\right), \quad v \sim N\left(0, \sigma_{v}^{2}\right)
$$

and

$$
\sigma_{u}=\left\{\frac{\Gamma(1+\beta) \sin \left(\frac{\pi \beta}{2}\right)}{\Gamma\left[\left(\frac{1+\beta}{2}\right)\right] \beta 2^{(\beta-1) / 2}}\right\}^{1 / \beta}, \sigma_{v}=1,
$$

where $\Gamma$ is a standard gamma function. The parameter $\beta$ is selected randomly between 0 and 2 . A high value of $\beta$ creates big jumps and increasing diversification, while low values lead to small jumps and increasing intensification. Figure 1 illustrates the mental search operator for two sample bids. As can be seen, the vicinity of a bid is searched with the aim of finding better bids. This process is performed for all bids in the population.

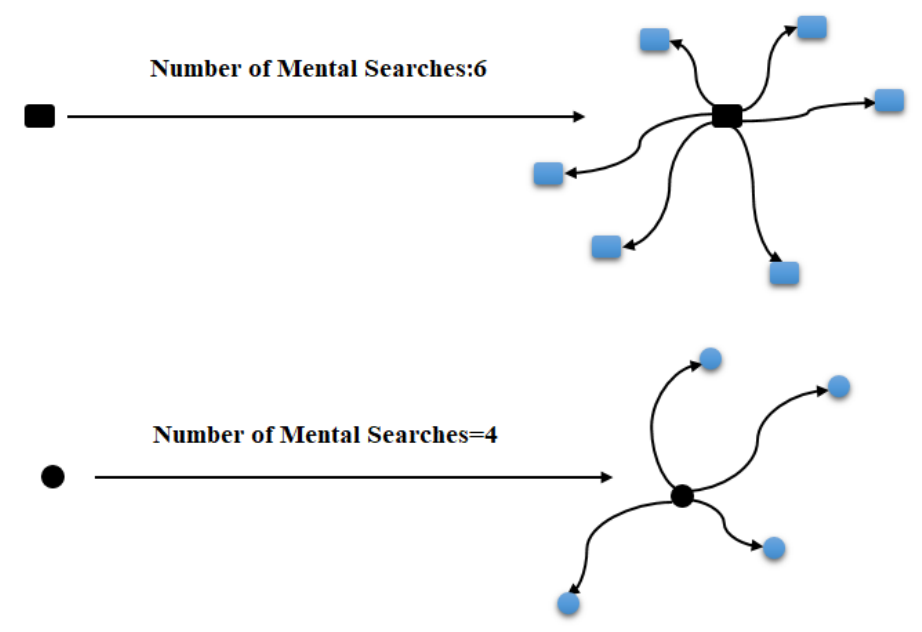

Figure 1: Examples of mental search operator.

\subsubsection{Grouping}

In the grouping operator, bids close together are located into one group. For grouping, a clustering algorithm is employed, in particular the $k$-means algorithm. After grouping, the mean cost function value for each group is calculated. The winner group is the group with the lowest mean cost function and thus represents a promising area. It is noteworthy that in order to find a promising area, a set of bids (and not the best available bids) are used, and thus a promising area may not necessarily contain the best bid available in the entire population. Figure 2 illustrates the grouping operator for a one-dimensional problem and shows that solutions close (similar) to each other are located in the same cluster. 


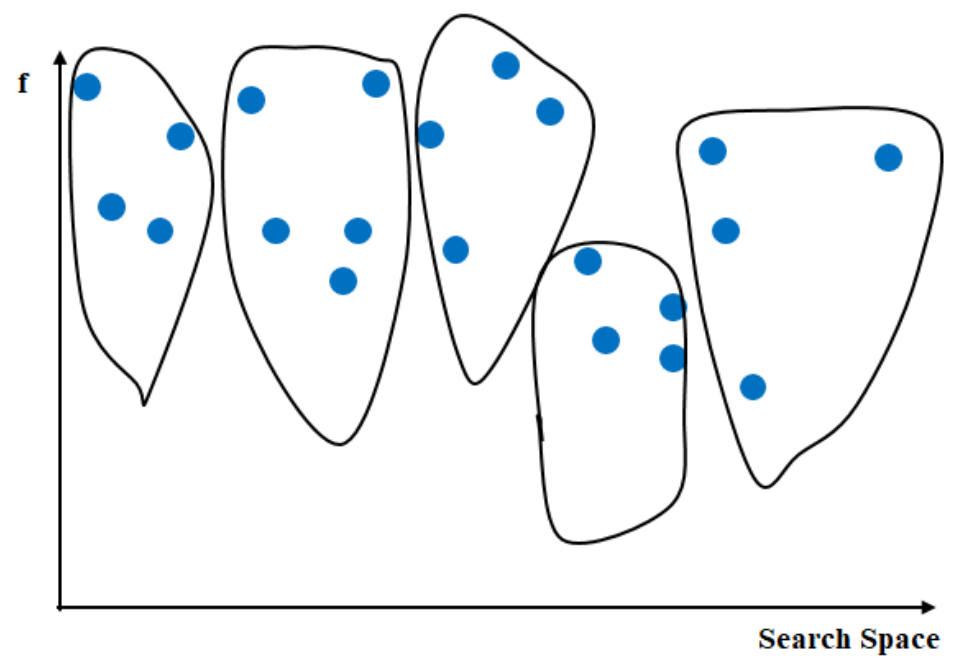

Figure 2: Example of bid grouping operator. The 21 bids are divided into 5 clusters.

\subsubsection{Moving towards the promising region}

In this stage, the other bids move towards the best bid in the promising area, i.e., the best bid of the winner cluster. Bids are updated as

$$
x_{n}^{t+1}=x_{n}^{t}+C\left(r \times \text { winner }_{n}^{t}-x_{n}^{t}\right),
$$

where $x_{n}^{t+1}$ is the $n$-th bid element at iteration $t+1$, winner ${ }_{n}^{t}$ is the $n$-th element of the best bid in the winner group, $t$ is the current iteration, $C$ is a constant number, and $r$ is a random number between 0 and 1 taken from the normal distribution.

\section{Automatic image clustering using local search-based HMS}

In this paper, we propose a novel approach to automatic clustering algorithm using a local search-based HMS algorithm for image segmentation. We first introduce the main components of our proposed ACLSHMS algorithm, and then explain the algorithm step-by-step.

\subsection{Representation of each bid}

The goal of our algorithm is to find the optimal number of clusters centres as well as the corresponding cluster centres. We assume that the maximum number of clusters $K_{\max }$ is known. Thus, ACLSHMS should find the appropriate number of clusters which is between 2 and $K_{\max }$.

In our approach, a bid is a real-valued vector of length $K_{\max }+K_{\max } \times d$. The first $K_{\max }$ elements are control "bits" in the interval $[0 ; 1]$ that are used to activate clusters. The remaining elements represent the $K_{\max }$ cluster centres of length of $d$. The overall structure of a bid is shown in Figure 3 where $m_{i}$ is the $i$-th cluster centre of length $d, d$ is the number of features, and $T_{i}$ is the control bit corresponding to cluster centre $m_{i}$. 


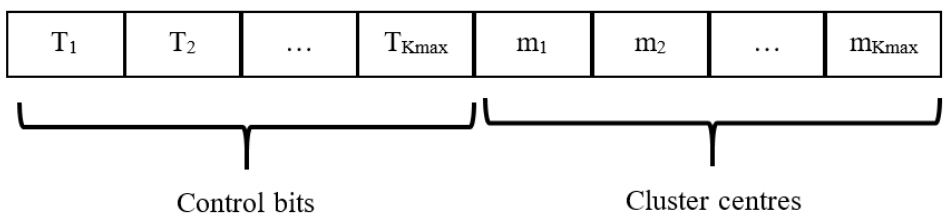

Figure 3: Structure of each bid in ACLSHMS.

The rule for selecting active clusters for a bid is

$$
\begin{array}{ll}
\text { if } T_{i}>0.5 \quad \text { then } m_{i} \text { is active } \\
\text { otherwise } m_{i} \text { is inactive. }
\end{array}
$$

Figure 4 is a representation of a sample bid for a two-dimensional problem with $K_{\max }=5$. Following the above rule, only 3 clusters have control bits higher than 0.5 and thus the three corresponding clusters (the red circles) are selected as active clusters.

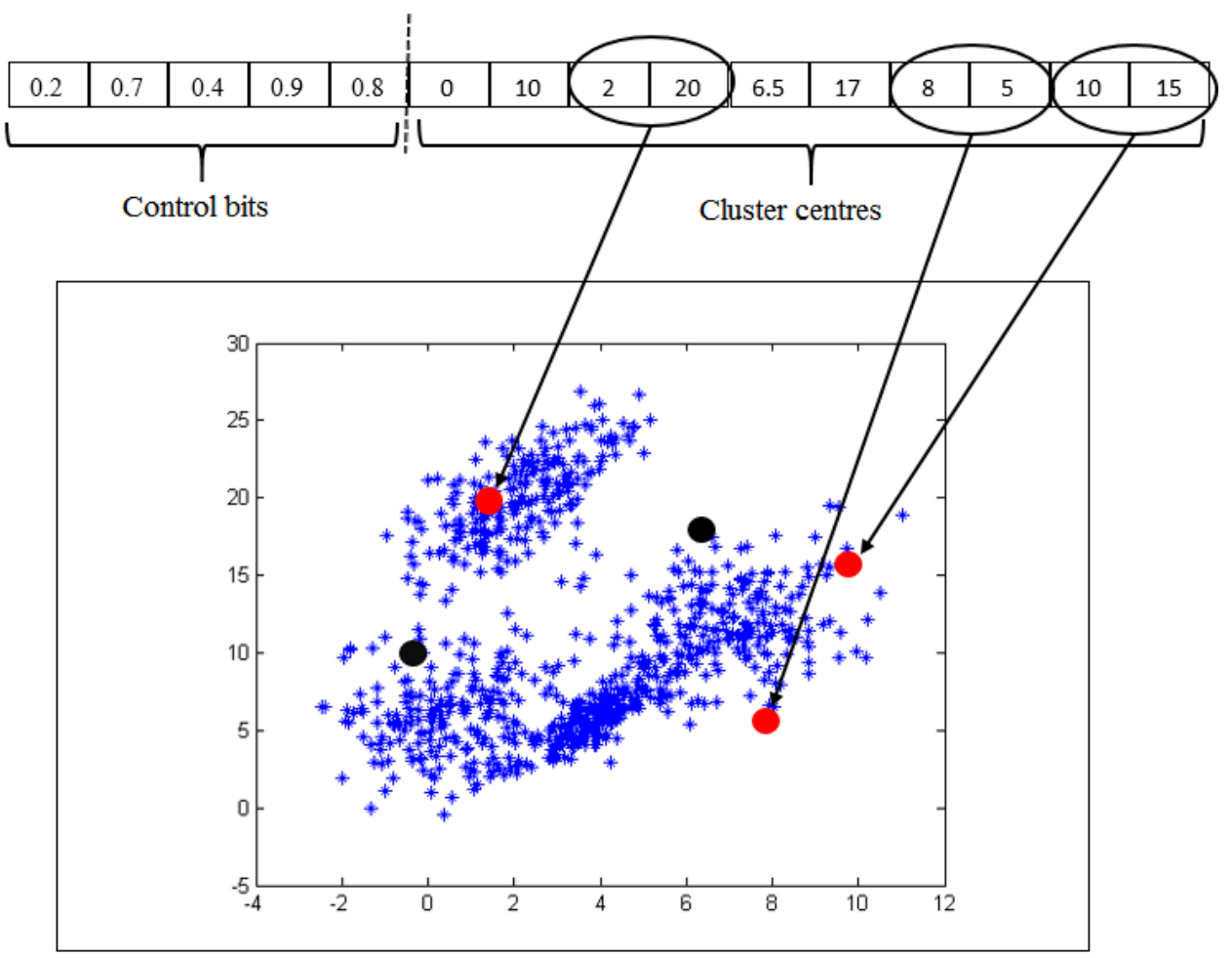

Figure 4: Example of a candidate solution (bid).

\subsection{Cost function}

Our employed cost function assigns a real valued number to each bid to specify the quality of the bid. For automatic clustering, we cannot use cost functions such as error, intra-cluster or inter-cluster distance that were previously used for static clustering [6] since this would generate a bias towards solutions with larger number of clusters. Hence, to evaluate a bid, we use a validation indicator that is more robust to the number of clusters. Validation indicators for clustering 
algorithms determine two aspects, namely compactness and separation. The samples in a cluster should be as much as possible similar to each other, and the variance of samples in each cluster can thus be used to represent the compactness of a cluster. On the other hand, clusters should be well-separated, and the distance between cluster centres can be used as a criterion for separation.

One of the most widely used clustering validation indicators is the Davies-Bouldin (DB) index [37] which measures the ratio of the sum of within cluster-scatter to between-cluster separation. The scatter within the $i$-th cluster is obtained as

$$
S_{i}=\frac{1}{n_{i}} \sum_{x_{j} \in c_{i}} d\left(x_{j}, m_{i}\right),
$$

where $n_{i}$ is the number of samples of the $i$-th cluster $c_{i}$, and $d\left(x_{j}, m_{i}\right)$ is the Euclidean distance between sample $x_{j}$ and its cluster centre $m_{i}$. The between-cluster separation is calculated as

$$
R_{i j}=\frac{S_{i}+S_{j}}{d\left(m_{i}, m_{j}\right)}, i \neq j
$$

and the DB index is defined as

$$
D B=\frac{1}{K} \sum_{k=1}^{K} R_{k},
$$

where $R_{k}=\max _{j=1,2, \ldots, K} R_{i j}$ and $i=1,2, \ldots, K$.

\subsection{Validation of cluster centres}

During calculation of the cost function, division by zero may occur if there are clusters with no or only one member. To avoid this problem, we check if such clusters exist and if so the cluster centres of these bids are re-initialised.

\subsection{Local search}

Local search tries to substitute the current candidate solution using a neighbour that improves the cost function, leading to increased intensification. The neighbourhood structure plays an important role for the efficacy of a local search algorithm; a candidate solution $S$ is called a neighbour $S$, so that $S$ is created by a small change in $S$.

In this paper, we employ a modified local search inspired from [38] to enhance the performance of our proposed algorithm. Our local search operator has four rules. For each bid, one of these rules is randomly selected, and several new bids are generated. Then, the best of these bids is selected to substitute the current bid. In the following we explain the four rules.

\subsubsection{Rule 1: change cluster centres}

In this rule, the value of an active cluster centre is substituted with a random value. Figure 5 illustrates this on an example of three active clusters. One of the clusters is randomly selected (in the example, the cluster with control bit 0.9 ), then the values defining the corresponding cluster centre corresponding are set to random values. The number of active clusters is not changed during this operation. 


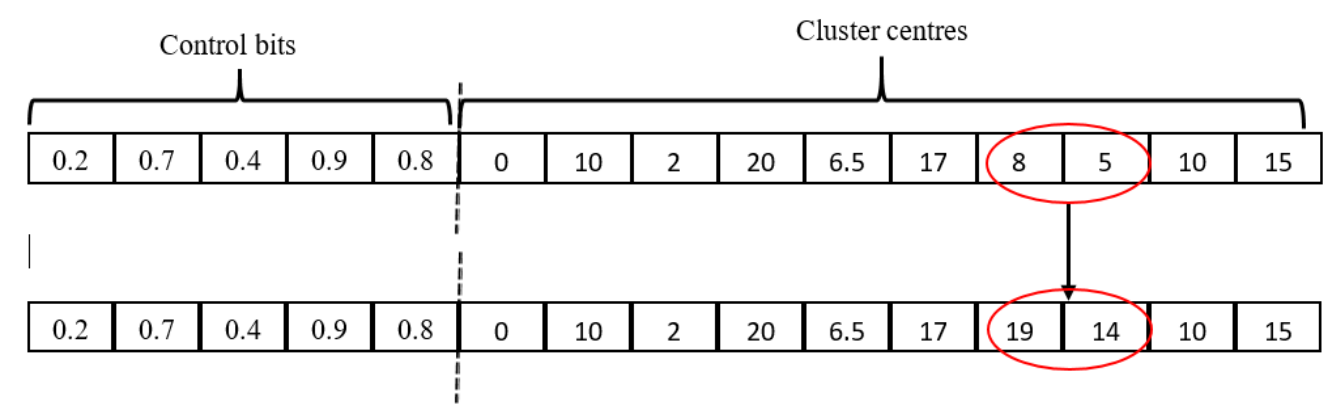

Figure 5: Example of changing cluster centre.

\subsubsection{Rule 2: change active clusters}

This rule changes the control bits of a bid. To this end, first an active cluster is randomly selected and made inactive, that is, the value of its control bit is substituted with a random number in [0;0.5]. Simultaneously, an inactive cluster is randomly selected and activated, i.e., its control bit set to a random number in $(0.5 ; 1]$. Figure 6 gives an example for this rule. The number of active clusters is not changed during this operation.

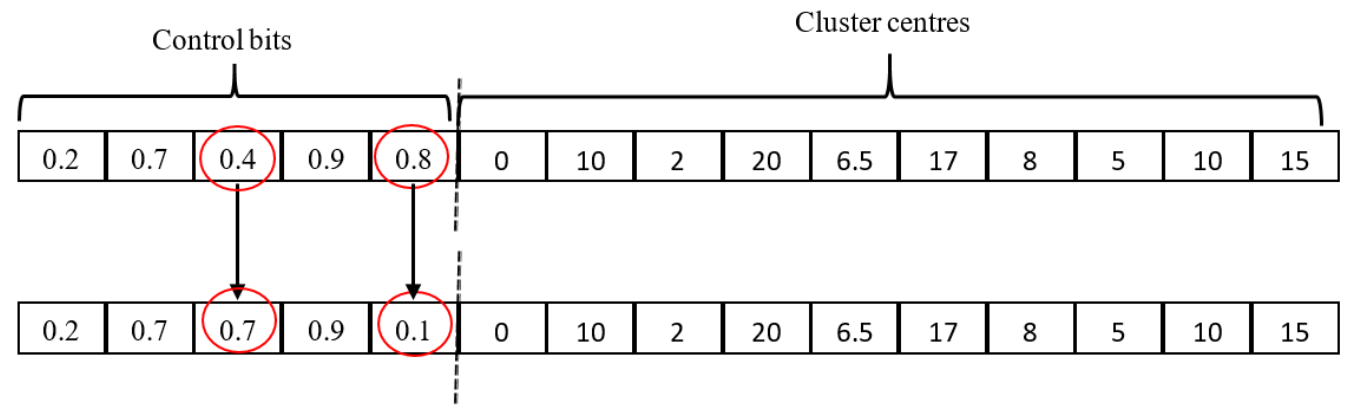

Figure 6: Example of changing active clusters.

\subsubsection{Rule 3: activating clusters}

The two rules above create local changes in the cluster centres and control bits but do not change the number of clusters. To support automatic clustering, some inactive clusters are randomly selected and activated, that is, their control bits set to a random number in $(0.5 ; 1]$. Figure 7 gives an example of this rule, where there are initially two inactive clusters with one of them being activated during the operation.

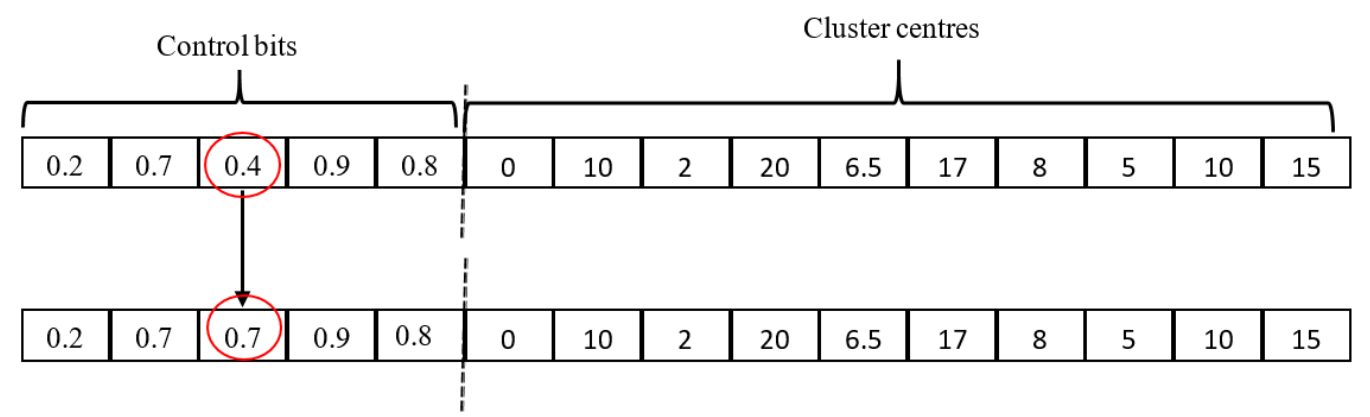

Figure 7: Example of activating clusters. 


\subsubsection{Rule 4: Deactivating clusters}

This operator is similar to the previous one except that a number of active clusters are randomly selected and deactivated by setting their control bits to random values in $[0 ; 0.5]$. An example is given in Figure 8 where two currently active clusters are deactivated.

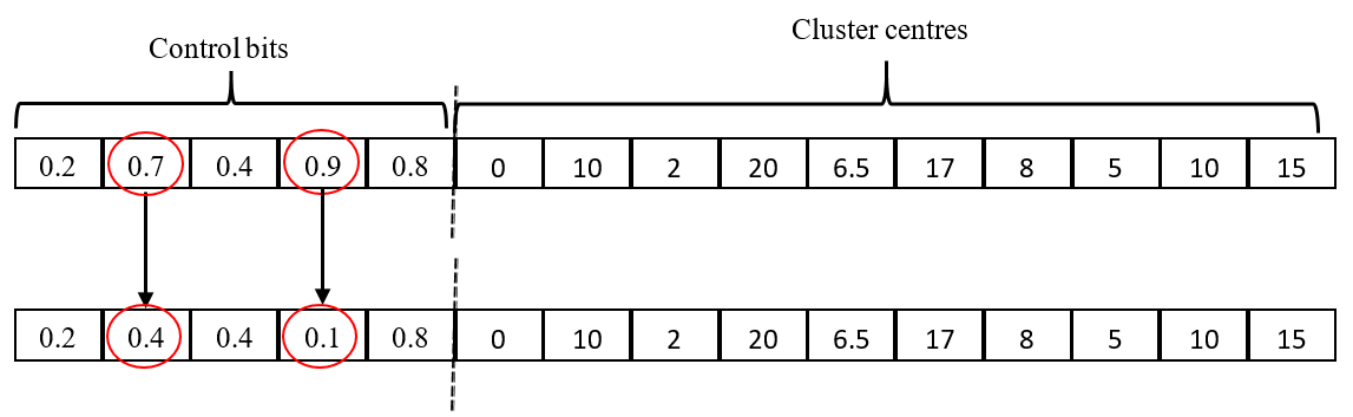

Figure 8: Example of deactivating clusters.

\subsection{General structure of proposed algorithm}

Our proposed ACLSHMS algorithm proceeds in the following steps:

Step 1: Parameter initialisation including the number of bids $N$, the number of clusters for bid grouping $K_{H M S}$, the minimum and maximum number of mental searches $M_{L}$ and $M_{L}$, and the number of new generated bids during local search Num.

Step 2: Create a random initial population of $N$ bids with each bid following the structure explained in Section 3.1

Step 3: Calculate the cost function for all bids using Eq. 10, validate the cluster centres, and choose the best bid $x^{*}$ in the current population.

Step 4: Select a random number in $\left[M_{L} ; M_{H}\right]$ for each bid representing its number of mental searches.

Step 5: Mental search operator: create new bids based on Levy distribution using Eqs. (1) and (3).

Step 6: Substitution operator: in case one of the new bids is better than the corresponding current one, it will substitute the latter.

Step 7: Bid grouping: cluster bids in search space using the $k$-means algorithm.

Step 8: Calculate the cost function for the members located in every group.

Step 9: Select the group with the lowest mean cost function value as the winner group representing a promising area.

Step 10: Move the other bids towards the best bid of the winner group using Eq. 6).

Step 11: Apply the local search operator from Section 3.4 on every bid.

Step 12: Find the best bid $x^{+}$from all current bids. If it is better than $x^{*}$, replace $x^{*}$ with it.

Step 13: If the stopping condition is not satisfied, go back to Step 4 


\section{Experimental results}

In this section, we study the efficacy of our proposed algorithm from different aspects. For this, we employ two types of greyscale images, real and synthetic images. Real images are used to assess the obtained cost function values for different algorithms, even though the actual number of clusters might not be known. In synthetic images, the number of clusters is determined in advance, and we can thus utilise them to assess whether a method can correctly identify the number of clusters or not. In total, we employ 17 commonly used images, shown in Figure 9 including Lenna, Airplane, House, Peppers, MRI, Caspian Sea, five images from the Berkeley database [39], namely 12003, 42049, 181079, 18054, and 385028 , and 6 synthetic images, $I 1$ to $I 6$.

We compare our proposed algorithm with other population-based clustering algorithms that have been previously applied for automatic image clustering, namely automatic clustering-based GA (ACGA) [40], automatic clustering-based differential evolution (ACDE) [25], automatic clustering-based particle swarm optimisation (ACPSO) [28], automatic clustering-based harmony search (ACHS) [29], and automatic clustering-based artificial bee colony (ACABC) [26], as well conventional clustering algorithms including $k$-means and fuzzy $c$-means. In addition, we compare our ACLSHMS algorithm to an algorithm that performs automatic clustering with HMS but without the local search (ACHMS).

The population size and the number of function evaluations (used as the stopping criterion) for all algorithms were set to 50 and 10000, respectively. Table 1 shows the values of the other parameters for the various algorithms. Due to the stochastic nature of the employed methods, each algorithm is run 25 times, and we report the average over these as the result.

Table 1: Parameter settings for all algorithms.

\begin{tabular}{llc}
\hline algorithm & parameter & value \\
\hline ACGA [9] & crossover probability & 0.8 \\
& mutation probability & $1 /$ length of a chromosome) \\
\hline ACDE [10] & scaling factor & 0.5 \\
& crossover probability & 0.1 \\
\hline ACPSO [41] & cognitive constant & 2 \\
& social constant & 2 \\
& inertia constant & 1 to 0 \\
\hline ACABC [42] & limit & $n_{e} \times$ dimensionality of problem \\
\hline ACHS [12] & harmony memory considering rate & 0.9 \\
& pitch adjusting rate & 0.1 \\
\hline ACHMS and ACLSHMS & number of clusters in bid grouping & 5 \\
& $C$ & 1 \\
\hline
\end{tabular}

The maximum number of clusters for all images, except $I 4, I 5$, and $I 6$, is set to 20 , and thus the length of each candidate solution is 40. An increase in the length of a bid leads to an expansion of the search space and as the latter becomes larger, an effective search is more difficult to conduct, a phenomenon that is known as the curse of dimensionality. Consequently, powerful operators are required to effectively search in high dimensions. To this end, we use the 3 more complex images, $I 4, I 5$, and $I 6$, to study the efficacy of the methods, and set the maximum number of clusters for these to 40 . 


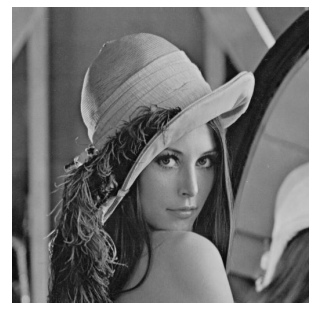

(a) Lenna

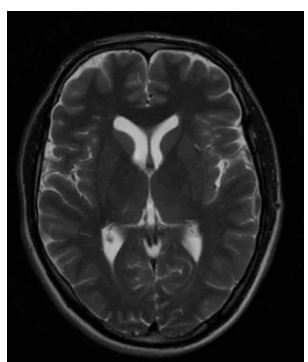

(e) MRI

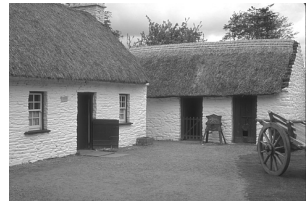

(i) 385028

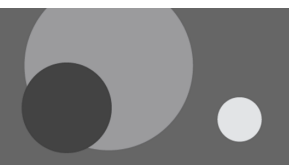

(m) I2

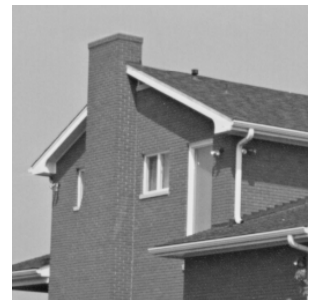

(b) House

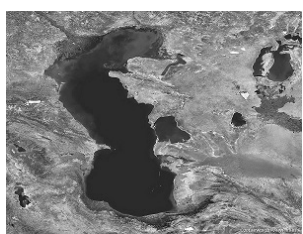

(f) Caspian Sea

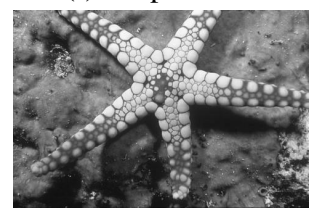

(j) 12003

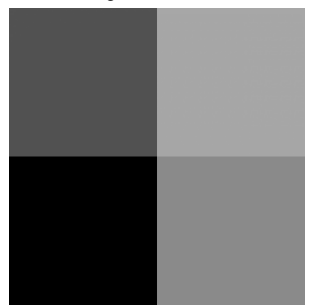

(n) I3

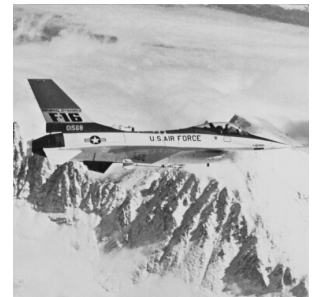

(c) Airplane

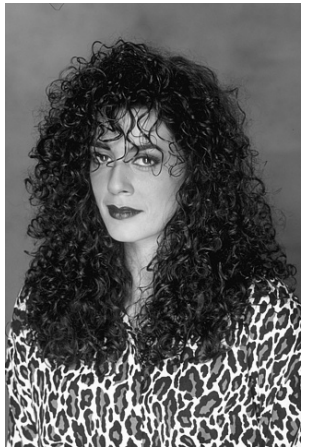

(g) 198054

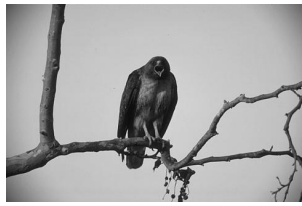

(k) 42049

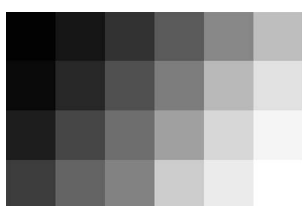

(o) I4

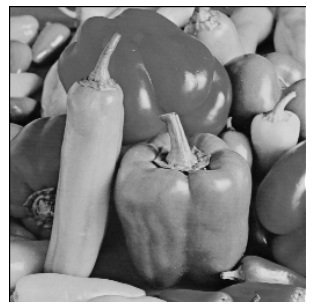

(d) Peppers

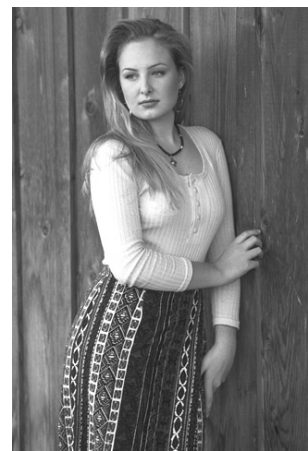

(h) 181079

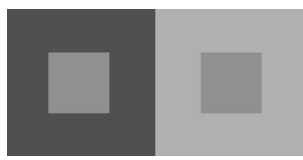

(1) I1

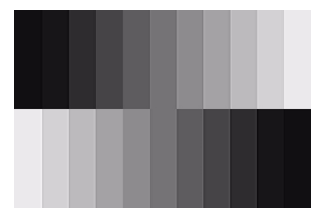

(p) I5

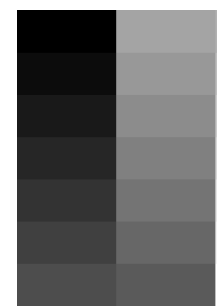

(q) I6

Figure 9: Test images. 


\subsection{Visual evaluation}

We first evaluate the visual performance of our approach compared to other population-based algorithms. For this, we select 3 of the images, 181079,385028 , and $I 6$, and show the segmented outputs (for other images, the results are similar) and compare these to manual segmentation results.

Figure 10 shows the results for image 181079. Since this image and 385028 are from the Berkeley segmentation database [39], there are several, often quite different, manual segmentations available. As can be seen, ACGA and ACPSO give relatively poor results, most noticeable in the background and the face of the girl. In contrast, both ACHMS and ACLSHMS yield a much better segmentation with ACLSHMS outperforming ACHMS also in the areas of the background and face.

Similar results can be observed for image 385028, for which the segmentations are given in Figure 11. Here, we can notice that in particular for the ground, ACHMS and ACLSHMS yield better results compared to the other algorithms. while furthermore ACLSHMS better segments the sky compared to ACHMS.

The results for image $I 6$ are given in Figure 12 Since it is a synthetic image, we also have a ground truth for the correct segmentation, and we can compare the number of obtained clusters in addition to inspecting the visual results. Again, ACHMS and ACLSHMS, even though not finding the perfect segmentation, clearly outperform the other approaches with ACLSHMS giving a slightly better result compared to ACHMS.

\subsection{Cost function evaluation}

The aim of this experiment is to evaluate our proposed method based on the cost function value and to compare it to the other methods. Table 2 gives the obtained results for all images and all algorithms and also reports the rank of each algorithm for each image and the resulting overall ranking over the whole image set.

As we can see from Table 2, for all images, except $I 5$ where it ranks third, ACLSHMS yields the lowest cost function value indicating the best automatic clustering result, which clearly demonstrates the usefulness of our proposed approach. Consequently, ACLSHMS clearly gives - and by a wide margin - the lowest average rank, and thus the best overall rank. Interestingly, the second ranked algorithm is ACHMS. This shows that our automatic clustering algorithm based on HMS is powerful, outperforming other methods even without the local search element. The third ranked algorithm is ACDE, followed by ACPSO and ACABC. Performing a pairwise comparison between algorithms, we can see that ACHMS outperforms ACGA, ACHS, and ACABC in all cases, and ACPSO in all but three, while ACDE gives better results for 5 of the 17 images. In turn, ACLSHMS outperforms ACGA, ACDE, ACHS, and ACABC on all images and ACPSO and ACHMS on all but one.

Images $I 4, I 5$, and $I 6$ can be used to evaluate the proposed algorithm in higher dimensions. As is evident from Table 2 ACLSHMS achieves the best result for $I 4$ and $I 6$ and second best for $I 5$, demonstrating the algorithm's capability to effectively deal with higher numbers of clusters and thus higher-dimensional search spaces. One of the main reasons for this effectiveness in higher dimensions is the application of a clustering algorithm which allows to find a promising region in search based on the information of each cluster. We can also notice that while ACGA performs worst for most images, here it yields better performance compared to ACABC and ACHS. 


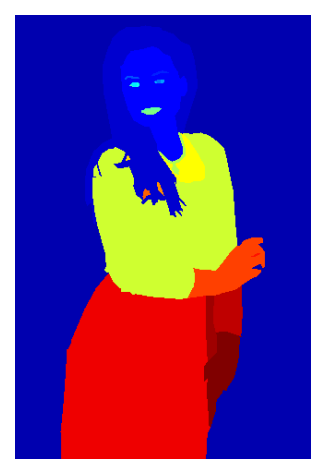

(a) manual segmentation 1

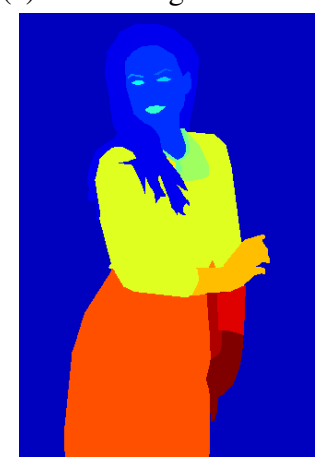

(d) manual segmentation 4

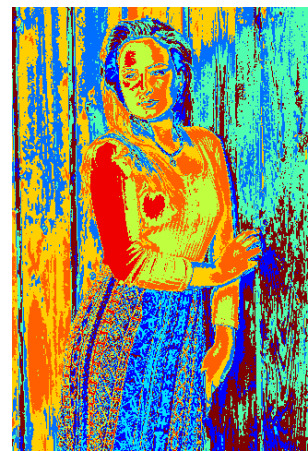

(g) ACGA

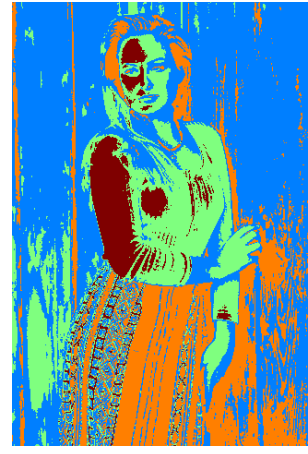

(j) $\mathrm{ACHS}$

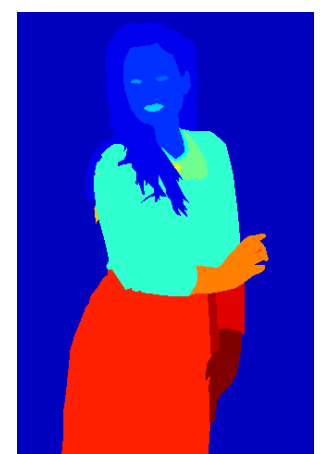

(b) manual segmentation 2

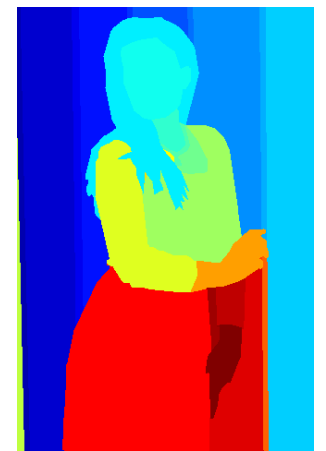

(e) manual segmentation 5

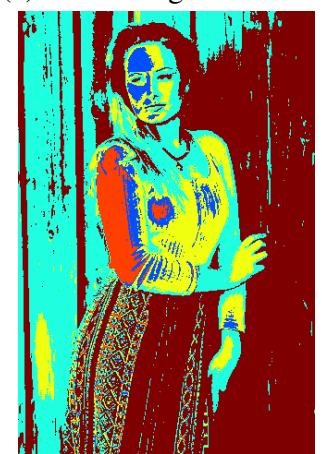

(h) $\mathrm{ACDE}$

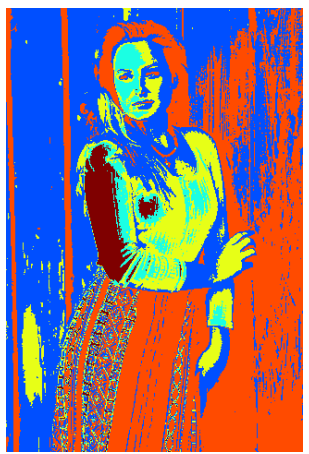

(k) $\mathrm{ACABC}$

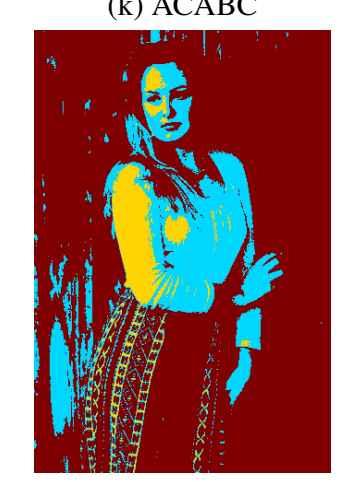

(m) ACLSHMS

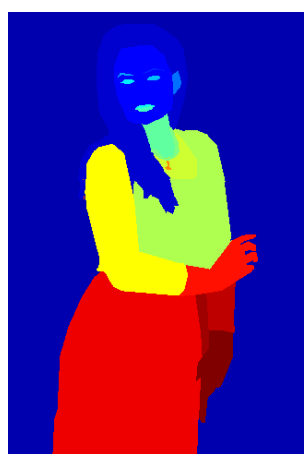

(c) manual segmentation 3

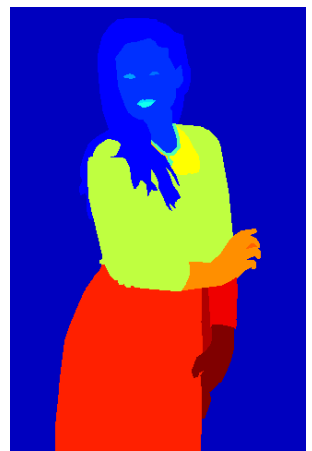

(f) manual segmentation 6

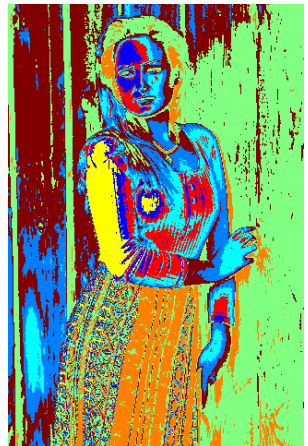

(i) ACPSO

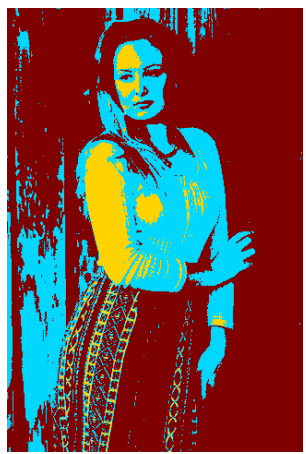

(1) ACHMS 


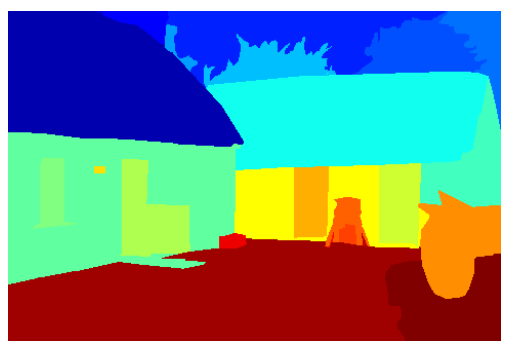

(a) manual segmentation 1

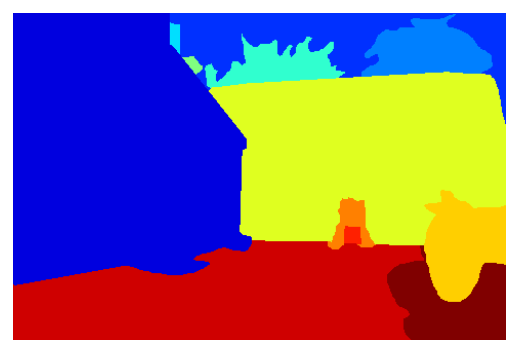

(b) manual segmentation 2

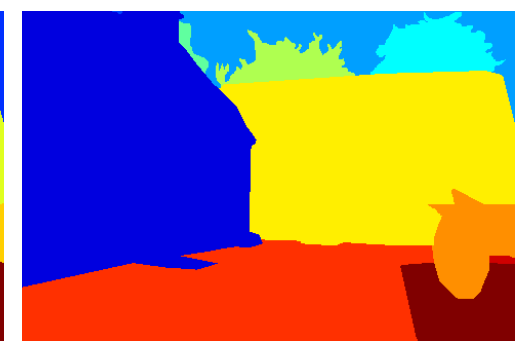

(c) manual segmentation 3

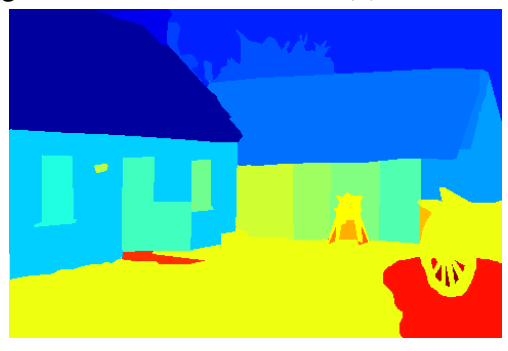

(d) manual segmentation 4

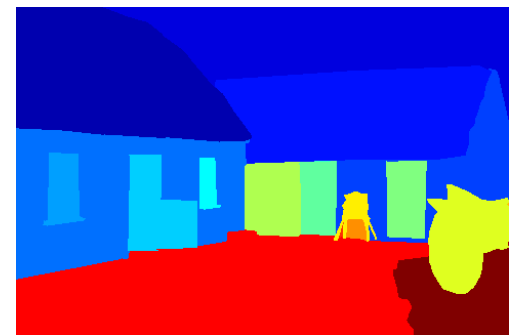

(e) manual segmentation 5

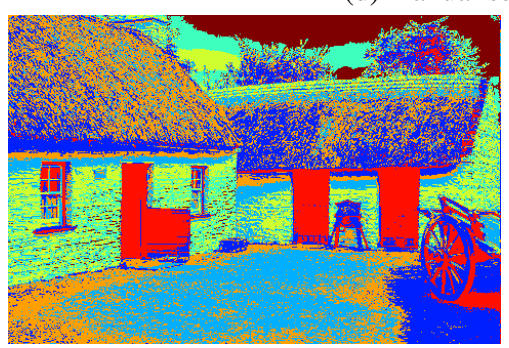

(f) ACGA

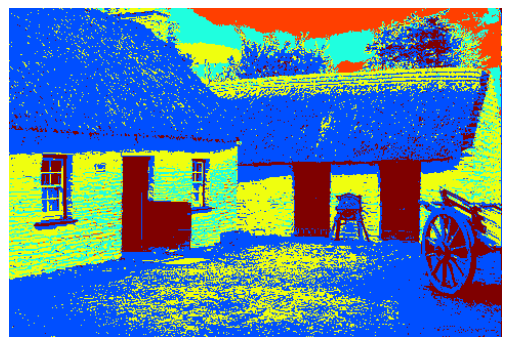

(i) ACHS

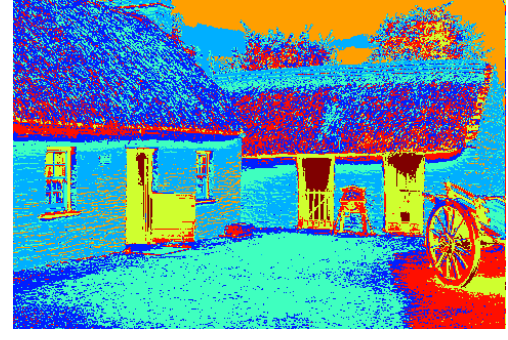

(g) ACDE

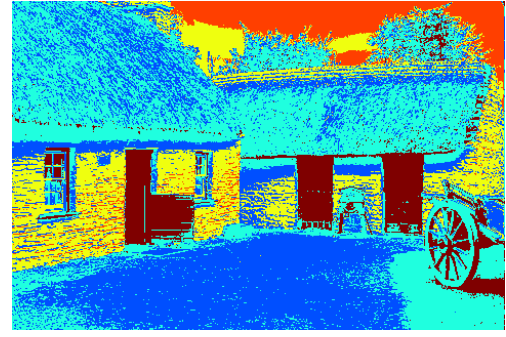

(j) $\mathrm{ACABC}$

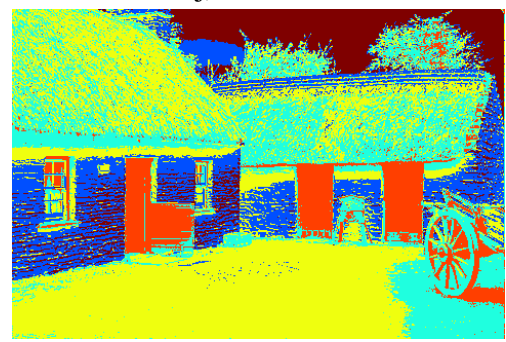

(1) ACLSHMS

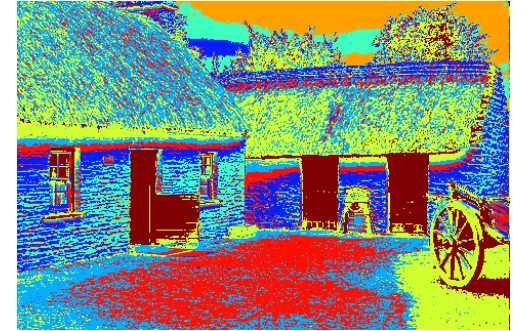

(h) ACPSO

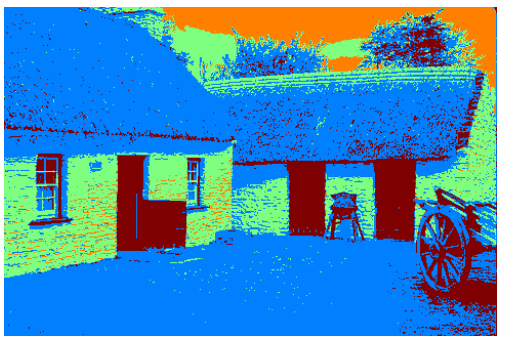

(k) ACHMS

Figure 11: Segmentation results for image 385028. 


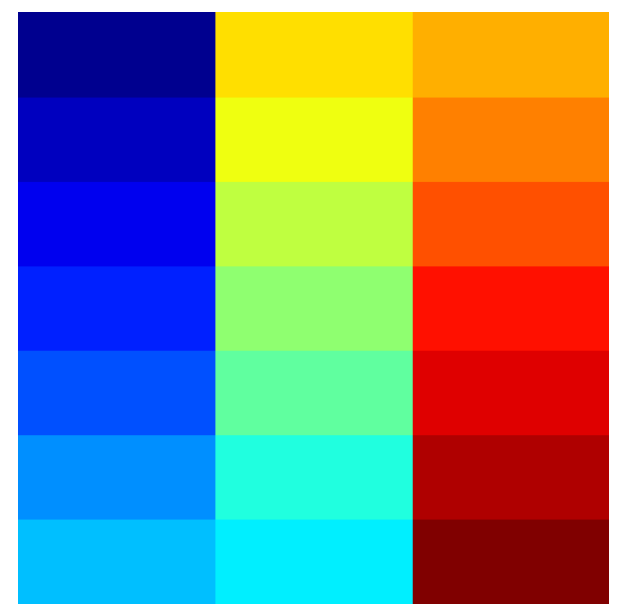

(a) ground truth segmentation

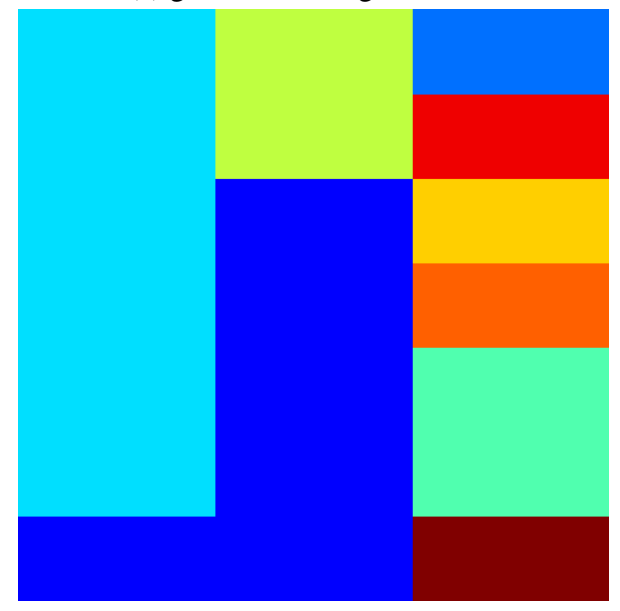

(c) ACDE

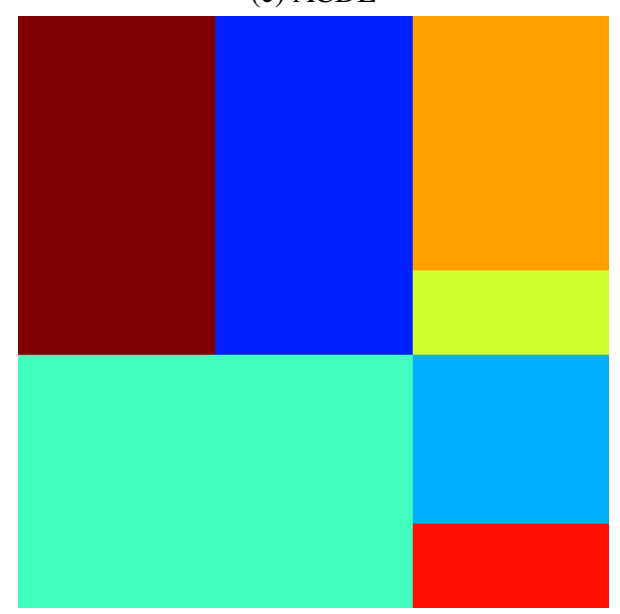

(e) ACHS

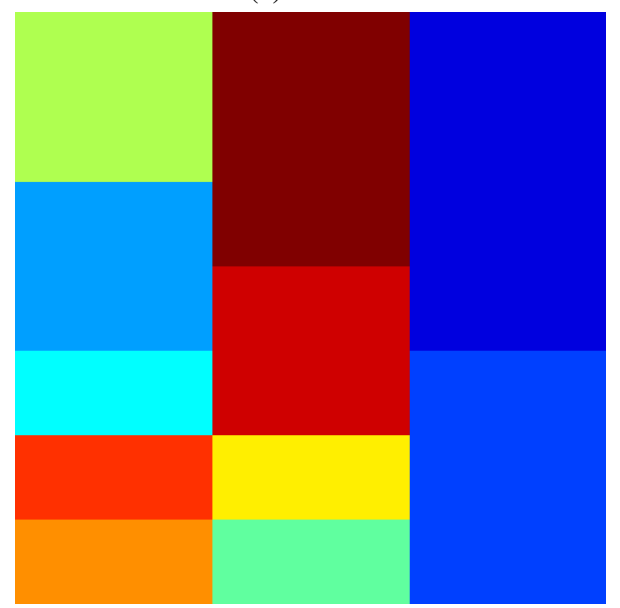

(g) ACHMS

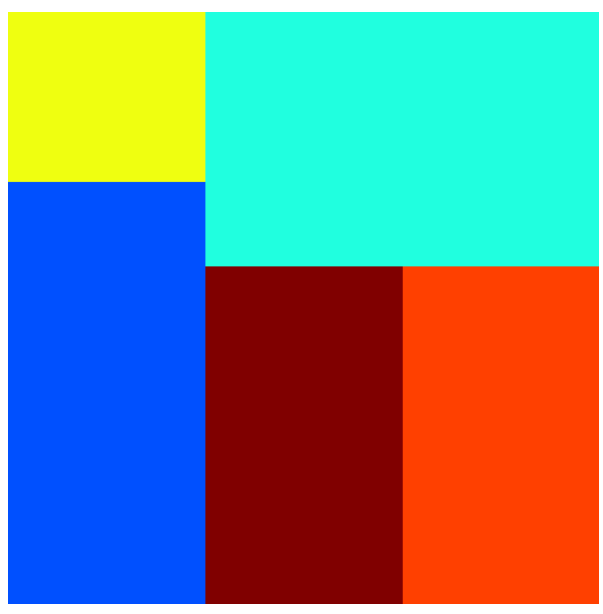

(b) ACGA

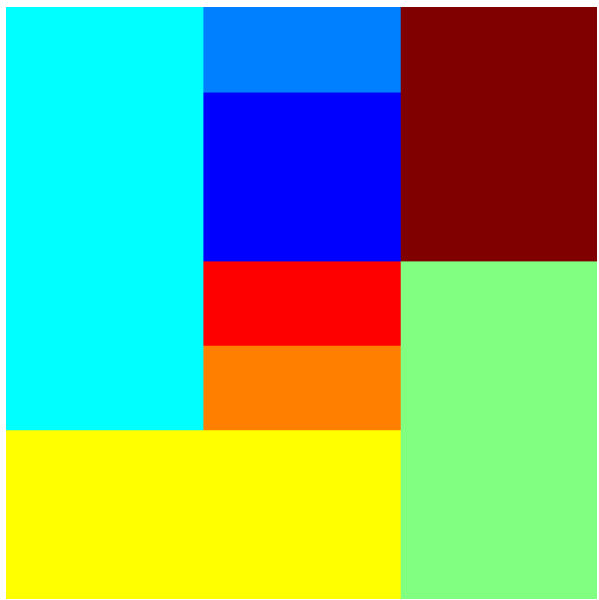

(d) ACPSO

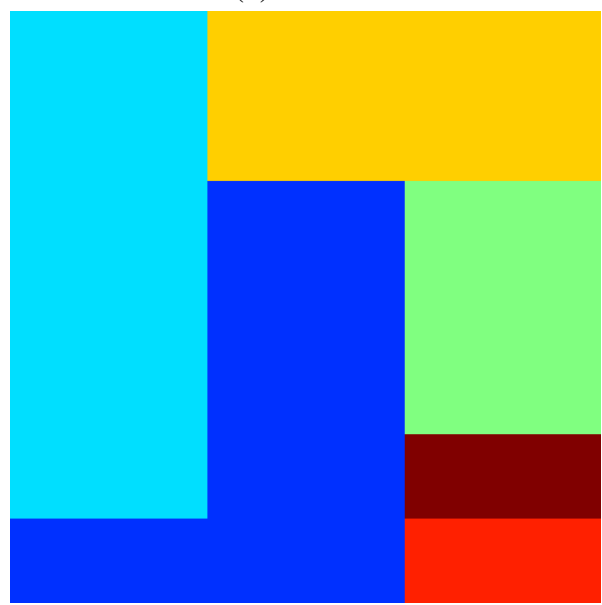

(f) $\mathrm{ACABC}$

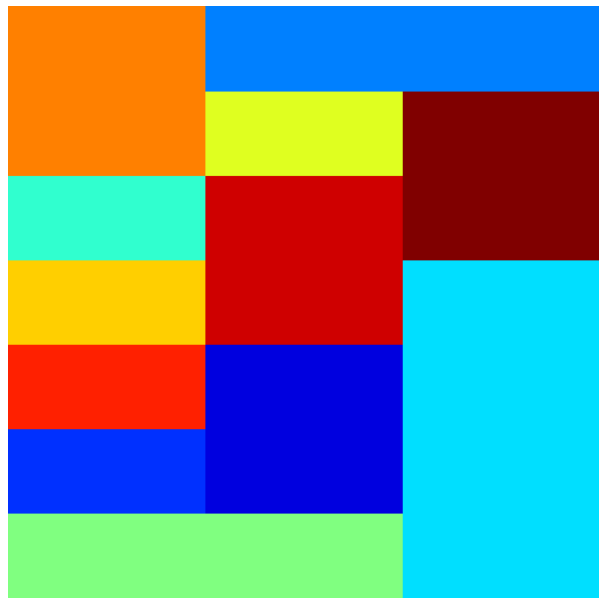

(h) ACLSHMS

Figure 12: Segmentation results for image $I 6$. 
Table 2: DB index results for all images and algorithms.

\begin{tabular}{|c|c|c|c|c|c|c|c|c|}
\hline image & & ACGA & ACDE & ACPSO & ACHS & $\mathrm{ACABC}$ & ACHMS & ACLSHMS \\
\hline \multirow[t]{2}{*}{ Lenna } & DB & 0.4408 & 0.4167 & 0.4245 & 0.4319 & 0.4302 & 0.4124 & 0.3964 \\
\hline & rank & 7 & 3 & 4 & 6 & 5 & 2 & 1 \\
\hline \multirow[t]{2}{*}{ Airplane } & DB & 0.4903 & 0.3495 & 0.3736 & 0.3758 & 0.3742 & 0.3544 & 0.3238 \\
\hline & rank & 7 & 2 & 4 & 6 & 5 & 3 & 1 \\
\hline \multirow[t]{2}{*}{ House } & DB & 0.4461 & 0.4002 & 0.4219 & 0.4106 & 0.4044 & 0.3916 & 0.3852 \\
\hline & rank & 7 & 3 & 6 & 5 & 4 & 2 & 1 \\
\hline \multirow[t]{2}{*}{ Peppers } & DB & 0.4314 & 0.4033 & 0.4053 & 0.4317 & 0.4220 & 0.3827 & 0.3639 \\
\hline & rank & 6 & 3 & 4 & 7 & 5 & 2 & 1 \\
\hline \multirow[t]{2}{*}{ MRI } & DB & 0.4212 & 0.3669 & 0.4080 & 0.4113 & 0.3781 & 0.3503 & 0.3426 \\
\hline & rank & 7 & 3 & 5 & 6 & 4 & 2 & 1 \\
\hline \multirow[t]{2}{*}{ Caspian Sea } & DB & 0.5555 & 0.4281 & 0.4321 & 0.4494 & 0.4429 & 0.4294 & 0.4129 \\
\hline & rank & 7 & 2 & 4 & 6 & 5 & 3 & 1 \\
\hline \multirow[t]{2}{*}{198054} & DB & 0.4193 & 0.3636 & 0.3909 & 0.4022 & 0.3724 & 0.3554 & 0.3459 \\
\hline & rank & 7 & 3 & 5 & 6 & 4 & 2 & 1 \\
\hline \multirow[t]{2}{*}{181079} & DB & 0.4401 & 0.4184 & 0.4335 & 0.4329 & 0.4299 & 0.4214 & 0.3969 \\
\hline & rank & 7 & 2 & 6 & 5 & 4 & 3 & 1 \\
\hline \multirow[t]{2}{*}{385028} & $\mathrm{DB}$ & 0.4502 & 0.4197 & 0.4224 & 0.4419 & 0.4414 & 0.4181 & 0.3966 \\
\hline & rank & 7 & 3 & 4 & 6 & 5 & 2 & 1 \\
\hline \multirow[t]{2}{*}{12003} & $\mathrm{DB}$ & 0.4535 & 0.4197 & 0.4241 & 0.4230 & 0.4273 & 0.4065 & 0.3933 \\
\hline & rank & 7 & 3 & 5 & 4 & 6 & 2 & 1 \\
\hline \multirow[t]{2}{*}{42049} & DB & 0.3440 & 0.3489 & 0.4075 & 0.3688 & 0.3634 & 0.3426 & 0.3373 \\
\hline & rank & 3 & 4 & 7 & 6 & 5 & 2 & 1 \\
\hline \multirow[t]{2}{*}{ I1 } & DB & 4.1210 & 0.0885 & 0.0854 & 0.1624 & 0.1393 & 0.1040 & 0.0687 \\
\hline & rank & 7 & 3 & 2 & 6 & 5 & 4 & 1 \\
\hline \multirow[t]{2}{*}{ I2 } & DB & 0.2002 & 0.0976 & 0.1977 & 0.1835 & 0.1649 & 0.1103 & 0.0654 \\
\hline & rank & 7 & 2 & 6 & 5 & 4 & 3 & 1 \\
\hline \multirow[t]{2}{*}{ I3 } & DB & 1.2659 & 0.1098 & 0.1353 & 0.2226 & 0.1348 & 0.0969 & 0.0433 \\
\hline & rank & 7 & 3 & 5 & 6 & 4 & 2 & 1 \\
\hline \multirow[t]{2}{*}{ I4 } & DB & 0.3729 & 0.3720 & 0.3713 & 0.4128 & 0.4000 & 0.3743 & 0.3406 \\
\hline & rank & 5 & 4 & 2 & 7 & 6 & 3 & 1 \\
\hline \multirow[t]{2}{*}{ I5 } & DB & 0.3089 & 0.3122 & 0.2258 & 0.3577 & 0.3654 & 0.2350 & 0.2382 \\
\hline & rank & 4 & 5 & 1 & 6 & 7 & 2 & 3 \\
\hline \multirow[t]{2}{*}{ I6 } & DB & 0.3663 & 0.3494 & 0.3477 & 0.4117 & 0.3930 & 0.3440 & 0.3257 \\
\hline & rank & 5 & 4 & 3 & 7 & 6 & 2 & 1 \\
\hline average rank & & 6.24 & 3.06 & 4.29 & 5.88 & 4.94 & 2.41 & 1.11 \\
\hline overall rank & & 7 & 3 & 4 & 6 & 5 & 2 & 1 \\
\hline
\end{tabular}

We also investigate the convergence rates for all algorithms and show the resulting convergence curves for some selected images in Figure 13 (the other images show similar characteristics). As can be seen from there, ACLSHMS yields the best convergence rate, while the local search in ACLSHMS leads to improved convergence behaviour compared to ACHMS.

Due to the non-deterministic nature of population-based metaheuristics, we also perform a statistical analysis of the obtained results, using the $p$-value to specify statistical significance. If the $p$-value is less than the significance level $\alpha$, then the null hypothesis $H_{0}$ is rejected, and the alternative hypothesis $H_{1}$ accepted. The null hypothesis here states that the algorithms do not differ statistically, while the alternative hypothesis indicates a statistical difference between the algorithms. We employ both the Friedman test and the Wilcoxon signed rank test [43] in our analysis.

The Friedman ranks for all algorithms are already given at the bottom of Table 2. As we have already discussed, ACLSHMS yields the lowest rank followed by ACHMS. According to the chi-square distribution table, the critical value for $7-1=6$ degrees of freedom and $\alpha=0.05$ is 12.592 . The obtained chi-square value is 70.34 and thus higher, 


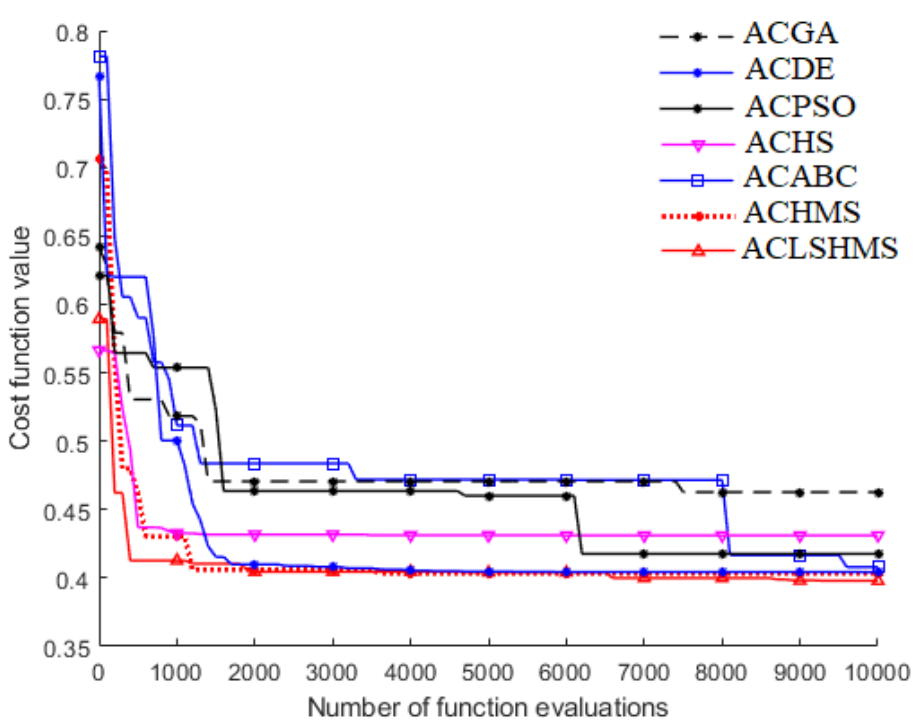

(a) Lenna

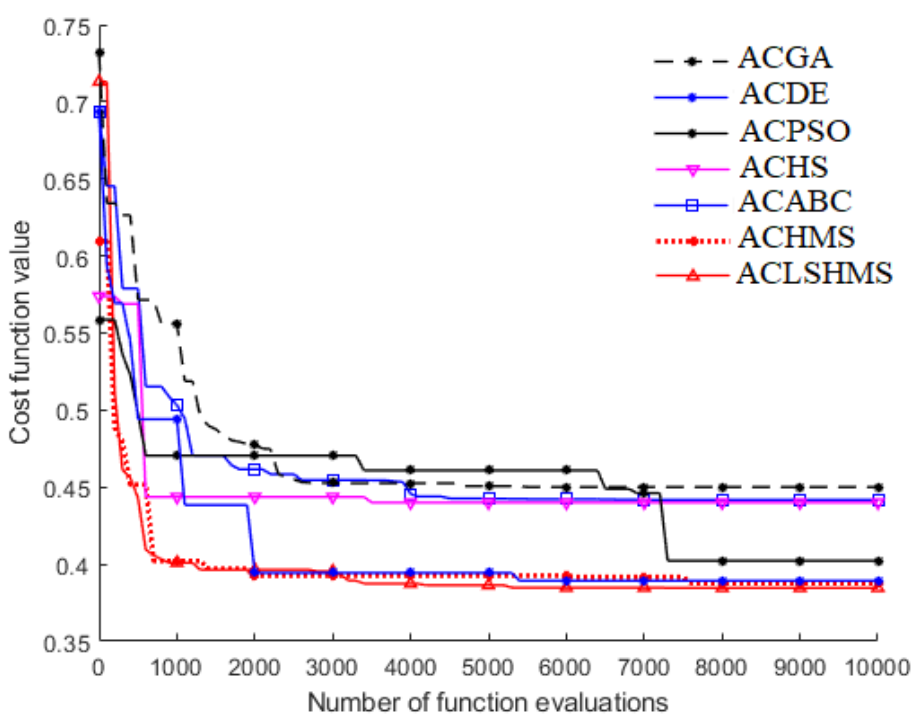

(b) Airplane

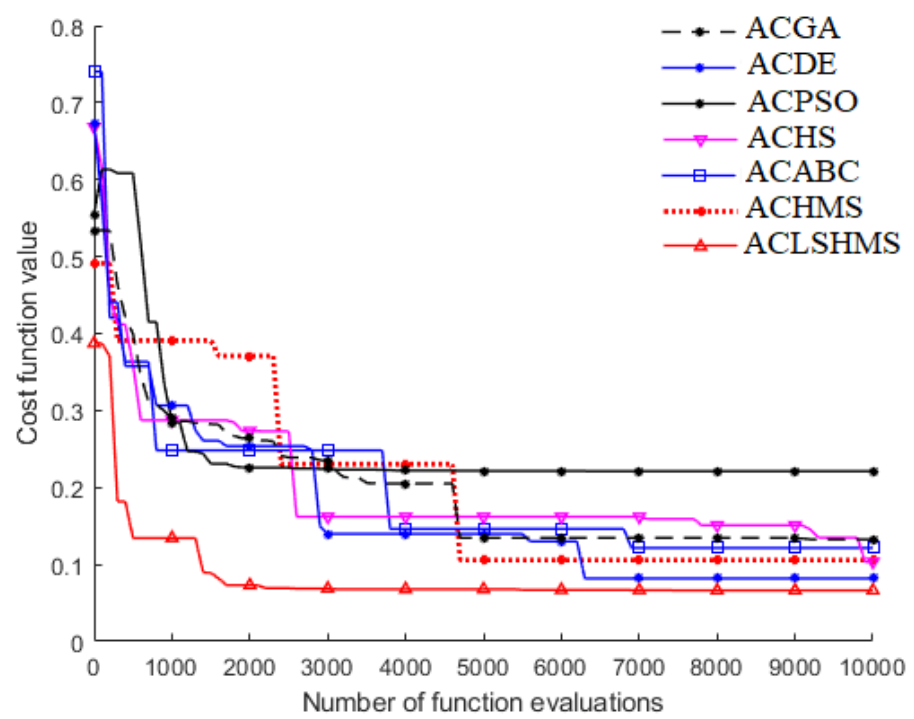

(c) I2

Figure 13: Convergence curves. 
signifying that hypothesis $H_{1}$ has been accepted. In other words, there is a statistical difference between the algorithms. A negligible $p$-value of 3.4886E-13 also means that hypothesis $H_{0}$ is rejected and $H_{1}$ is confirmed.

The Wilcoxon signed rank test allows us to investigate if there is a statistical difference between ACLSHMS and any of the other algorithms. Table 3 lists the obtained results for this. As we note, in all cases the $p$-value is less than 0.05 which indicates that ACLSHMS is statistically superior to all other algorithms.

Table 3: Wilcoxon signed rank test results.

\begin{tabular}{lc}
\hline comparison & $p$-value \\
\hline ACLSHMS vs. ACGA & $2.9305 \mathrm{E}-04$ \\
ACLSHMS vs. ACDE & $2.9305 \mathrm{E}-04$ \\
ACLSHMS vs. ACPSO & $3.5153 \mathrm{E}-04$ \\
ACLSHMS vs. ACHS & $2.9305 \mathrm{E}-04$ \\
ACLSHMS vs. ACABC & $2.9305 \mathrm{E}-04$ \\
\hline ACLSHMS vs. ACHMS & $3.5153 \mathrm{E}-04$ \\
\hline
\end{tabular}

\subsection{Stability analysis}

Since, due to the stochastic nature of the algorithms, the output of each run will be different, we further assess the stability of the methods by means of the standard deviation defined as $\sigma=\sqrt{\sum_{i=1}^{M} \frac{\left(x_{i}-\mu\right)^{2}}{M}}$, where $M$ is the number of independent runs ( $M=25$ in our experiments), $x_{i}$ is the value of the cost function in $i$-th run, and $\mu$ is the mean cost function (i.e., the results from Table 27. A lower $\sigma$ indicates a more robust algorithm. Table 4 gives the standard deviations for all algorithms and all images. As we can see from there, for most images (13 out of 17), ACLSHMS gives the lowest standard deviation, demonstrating the algorithm's robustness.

Table 4: Standard deviation results for all images and algorithms. The best result for each image is bolded.

\begin{tabular}{llllllll}
\hline image & ACGA & ACDE & ACPSO & ACHS & ACABC & ACHMS & ACLSHMS \\
\hline Lenna & 0.0259 & 0.0131 & 0.0311 & 0.0215 & 0.0191 & 0.0117 & $\mathbf{0 . 0 0 2 8}$ \\
Airplane & 0.0709 & 0.0121 & 0.0322 & 0.0197 & 0.0130 & 0.0136 & $\mathbf{0 . 0 0 5 0}$ \\
House & 0.0240 & 0.0125 & 0.0226 & 0.0138 & 0.0093 & 0.0046 & $\mathbf{0 . 0 0 1 6}$ \\
Peppers & 0.0405 & 0.0180 & 0.0320 & 0.0366 & 0.0270 & 0.0092 & $\mathbf{0 . 0 0 1 6}$ \\
MRI & 0.0389 & 0.0217 & 0.0275 & 0.0317 & 0.0201 & 0.0097 & $\mathbf{0 . 0 0 1 6}$ \\
Caspian Sea & 0.1074 & 0.0125 & 0.0198 & 0.0162 & 0.0189 & 0.0101 & $\mathbf{0 . 0 0 5 6}$ \\
198054 & 0.0388 & 0.0155 & 0.0343 & 0.0281 & 0.0194 & 0.0060 & $\mathbf{0 . 0 0 3 2}$ \\
181079 & 0.0164 & 0.0112 & 0.0411 & 0.0189 & 0.0156 & 0.0104 & $\mathbf{0 . 0 0 8 1}$ \\
385028 & 0.0278 & 0.0173 & 0.0249 & 0.0140 & $\mathbf{0 . 0 0 9 8}$ & 0.0172 & 0.0135 \\
12003 & 0.0372 & 0.0172 & 0.0236 & 0.0379 & 0.0128 & 0.0101 & $\mathbf{0 . 0 0 1 7}$ \\
42049 & 0.0043 & 0.0090 & 0.0428 & 0.0188 & 0.0316 & 0.0027 & $\mathbf{0 . 0 0 2 9}$ \\
I1 & 0.3155 & 0.0095 & 0.0237 & 0.0343 & 0.0248 & 0.0180 & $\mathbf{0 . 0 0 4 5}$ \\
I2 & 0.1005 & 0.0161 & 0.0971 & 0.0450 & 0.0301 & 0.0204 & $\mathbf{0 . 0 0 1 5}$ \\
I3 & 0.7952 & 0.0284 & 0.0928 & 0.0447 & 0.0384 & 0.0370 & $\mathbf{0 . 0 3 4 4}$ \\
I4 & 0.0391 & 0.0178 & 0.0341 & 0.0173 & $\mathbf{0 . 0 1 5 1}$ & 0.0162 & 0.0252 \\
I5 & 0.0449 & 0.0163 & 0.0369 & 0.0246 & 0.0256 & $\mathbf{0 . 0 2 0 7}$ & 0.0218 \\
I6 & 0.0613 & 0.0266 & 0.0459 & 0.0231 & $\mathbf{0 . 0 1 5 8}$ & 0.0196 & 0.0275 \\
\hline
\end{tabular}

\subsection{Number of clusters}

One of the main characteristics of the proposed algorithm is that no prior knowledge on the number of clusters is required. We therefore perform an evaluation that investigates the number of clusters that are obtained by our and by competitor 
algorithms. For this, we consider three types of images:

1. Popular test images such as it Lenna: here, the number of correct clusters is not known;

2. Images from the Berkeley Segmentation database: these come with hand-labelled segmentations. However, there are multiple, often quite differing, segmentations for each image and thus segmentations with different numbers of clusters;

3. Synthetic images: the exact number of clusters is known.

For images such as Lenna, we cannot directly compare the algorithms in terms of the obtained numbers of clusters. Although we list the results for these in Table 5, we focus in the following on the other two image types which allow for a more precise evaluation.

Table 5: Average numbers of clusters obtained for popular test images.

\begin{tabular}{lccccccc}
\hline image & ACGA & ACDE & ACPSO & ACHS & ACABC & ACHMS & ACLSHMS \\
\hline Lenna & 6.60 & 3.68 & 7.00 & 3.60 & 3.48 & 3.12 & 3.44 \\
Airplane & 6.72 & 4.12 & 6.52 & 4.00 & 3.88 & 4.24 & 4.04 \\
House & 8.00 & 2.88 & 7.16 & 2.76 & 2.72 & 2.88 & 3.16 \\
Peppers & 6.36 & 4.04 & 6.36 & 4.44 & 4.20 & 3.00 & 3.00 \\
MRI & 7.24 & 3.56 & 7.56 & 3.80 & 3.24 & 3.08 & 3.00 \\
Caspian Sea & 8.00 & 5.40 & 7.84 & 4.20 & 4.60 & 5.28 & 5.20 \\
\hline
\end{tabular}

As mentioned, for images from the Berkeley database, there are multiple segmentations with typically different numbers of clusters. We define an error measure as the minimum difference between the number of clusters and the correct number of clusters

$$
E_{\min }=\min _{i}\left\{\left|t-o_{i}\right|\right\}
$$

where $t$ is the obtained number of clusters, and $o_{i}$ is the correct number of clusters for $i$-th hand-labelled segmentation. Table 6 gives the results for this, from where we can see that ACLSHMS achieves the lowest error for all but one image, demonstrating the automatic clustering ability of our proposed algorithm. We also notice that the local search element in ACLSHMS leads to improved performance compared to ACHMS.

Table 6: Obtained numbers of clusters $N$ and errors $E_{\min }$ for images from the Berkeley database. The best error result for each image is bolded.

\begin{tabular}{lcccccccc}
\hline image & & ACGA & ACDE & ACPSO & ACHS & ACABC & ACHMS & ACLSHMS \\
\hline 198054 & $E_{\text {min }}$ & 10.04 & 9.60 & 10.04 & 10.84 & $\mathbf{9 . 4 8}$ & 9.84 & 9.60 \\
& $N$ & 2.96 & 3.40 & 2.96 & 2.16 & 3.52 & 3.16 & 3.40 \\
181079 & $E_{\text {min }}$ & 14.33 & 13.08 & 14.92 & 14.24 & 14.28 & 13.72 & $\mathbf{1 3 . 6 4}$ \\
& $N$ & 3.67 & 4.92 & 3.08 & 3.76 & 3.72 & 4.28 & 4.36 \\
385028 & $E_{\text {min }}$ & 6.60 & 6.68 & 6.56 & 6.68 & 6.84 & 6.28 & $\mathbf{6 . 0 4}$ \\
& $N$ & 4.40 & 4.32 & 4.44 & 4.32 & 4.16 & 3.72 & 4.96 \\
12003 & $E_{\text {min }}$ & 3.40 & 4.32 & 3.44 & 3.44 & 2.88 & 2.92 & $\mathbf{2 . 5 2}$ \\
& $N$ & 2.60 & 1.68 & 2.56 & 2.56 & 3.12 & 3.08 & 3.48 \\
42049 & $E_{\min }$ & 15.00 & 14.80 & 14.88 & 15.12 & 15.00 & 15.04 & $\mathbf{1 4 . 0 8}$ \\
& $N$ & 2.00 & 2.20 & 2.12 & 1.88 & 2.00 & 1.96 & 2.92 \\
\hline
\end{tabular}

For synthetic images, the correct numbers of clusters are known, and an error measure can hence be simply defined 
by

$$
E=|t-o|
$$

where $t$ is the obtained number of clusters, and $o$ is the correct number of clusters. The results for this are shown in Table 7, which indicates that our ACLSHMS algorithm gives the best results for all but image $I 3$, while for the latter ACHMS yields the lowest error. In particular, for the images with more regions, 14 to I6, ACLSHMS gave the best results which confirms its effectiveness in high dimensional search spaces. Comparing ACHMS and ACLSHMS, we can also see that the local search clearly leads to improved performance.

Table 7: Obtained numbers of clusters $N$ and errors $E$ for synthetic images. The best error result for each image is bolded.

\begin{tabular}{lcccccccc}
\hline image & & ACGA & ACDE & ACPSO & ACHS & ACABC & ACHMS & ACLSHMS \\
\hline I1 & $E$ & 2.96 & 0.24 & 3.80 & 0.64 & 0.20 & 0.48 & $\mathbf{0 . 1 2}$ \\
& $N$ & 5.96 & 3.24 & 6.80 & 3.64 & 3.20 & 3.48 & 3.92 \\
I2 & $E$ & 1.92 & $\mathbf{0 . 0 0}$ & 1.84 & 0.08 & 0.04 & $\mathbf{0 . 0 0}$ & $\mathbf{0 . 0 0}$ \\
& $N$ & 5.92 & 4.00 & 5.84 & 4.08 & 4.04 & 4.00 & 4.00 \\
I3 & $E$ & 1.00 & 1.72 & 1.76 & 1.94 & 1.92 & $\mathbf{1 . 6 8}$ & 1.80 \\
& $N$ & 5.00 & 5.72 & 4.76 & 5.24 & 5.52 & 5.68 & 5.80 \\
I4 & $E$ & 16.00 & 15.56 & 15.60 & 18.12 & 18.76 & 15.48 & $\mathbf{1 3 . 5 2}$ \\
& $N$ & 8.00 & 8.44 & 8.40 & 5.88 & 5.24 & 8.52 & 10.48 \\
I5 & $E$ & 4.00 & 2.44 & 2.20 & 2.84 & 2.84 & 1.76 & $\mathbf{1 . 2 4}$ \\
& $N$ & 7.00 & 8.56 & 8.60 & 8.16 & 8.16 & 9.24 & 9.76 \\
I6 & $E$ & 13.48 & 11.20 & 12.68 & 13.04 & 15.32 & 10.64 & $\mathbf{1 0 . 3 6}$ \\
& $N$ & 7.52 & 9.80 & 8.32 & 7.96 & 5.68 & 10.36 & 10.64 \\
\hline
\end{tabular}

\subsection{Comparison with conventional clustering-based methods}

In addition to the extensive comparison of our proposed algorithm against other automatic image clustering algorithms from the literature, we also evaluate our approach against conventional clustering-based methods including $k$-means and fuzzy $c$-means. Since these are not automatic, here we set the number of clusters to the same number of clusters of the metaheuristic that offered the best result. For example, for Lenna it is set to 3 since ACLSHMS gave the best results for that image and the average number of clusters over the 25 runs was 3.44 which is rounded to 3 . As above, we employ the DB index for comparison. Table 8 shows the obtained results in terms of mean and standard deviation over 25 independent runs. As can be observed, in all cases ACLSHMS yields significantly lower cost function values compared to $k$-means and FCM, while also giving lower standard deviation results for all images indicating both better performance and better robustness. In addition, the results of a Wilcoxon signed rank, given in Table 9, confirm that our proposed methods are statistically significantly better.

\subsection{Image segmentation performance}

Last but not least, we evaluate our algorithm in terms of image segmentation quality and compare it to the other methods. To this end, we employ five image segmentation criteria, including both unsupervised and supervised measures, from the literature, namely:

- Borsotti criterion (BOR) [44]: an unsupervised criterion based on the number, variance, and the areas of image regions. A lower BOR value indicates better performance; 
Table 8: DB index results for HMS-based and conventional clustering-based algorithms. The best results for each image are bolded.

\begin{tabular}{lcccccccc}
\hline \multirow{2}{*}{ image } & \multicolumn{2}{c}{$k$-means } & \multicolumn{2}{c}{ FCM } & \multicolumn{2}{c}{ ACHMS } & \multicolumn{2}{c}{ ACLSHMS } \\
\cline { 2 - 9 } & mean & stddev & mean & stddev & mean & stddev & mean & stddev \\
\hline Lenna & 0.5705 & 0.0526 & 0.5762 & 0.0465 & 0.4124 & 0.0117 & $\mathbf{0 . 3 9 6 4}$ & $\mathbf{0 . 0 0 2 8}$ \\
Airplane & 0.4297 & 0.0249 & 0.4189 & 0.0291 & 0.3544 & 0.0136 & $\mathbf{0 . 3 2 3 8}$ & $\mathbf{0 . 0 0 5 0}$ \\
House & 0.5736 & 0.0423 & 0.6279 & 0.0338 & 0.3916 & 0.0046 & $\mathbf{0 . 3 8 5 2}$ & $\mathbf{0 . 0 0 1 6}$ \\
Peppers & 0.4980 & 0.0542 & 0.4985 & 0.0529 & 0.3827 & 0.0092 & $\mathbf{0 . 3 6 3 9}$ & $\mathbf{0 . 0 0 1 6}$ \\
MRI & 0.4455 & 0.0410 & 0.4319 & 0.0484 & 0.3503 & 0.0097 & $\mathbf{0 . 3 4 2 6}$ & $\mathbf{0 . 0 0 1 6}$ \\
Caspian Sea & 0.5410 & 0.0304 & 0.5181 & 0.0252 & 0.4294 & 0.0101 & $\mathbf{0 . 4 1 2 9}$ & $\mathbf{0 . 0 0 5 6}$ \\
42049 & 0.4455 & 0.0535 & 0.4395 & 0.0525 & 0.3554 & 0.0060 & $\mathbf{0 . 3 4 5 9}$ & $\mathbf{0 . 0 0 3 2}$ \\
12003 & 0.5429 & 0.0520 & 0.5202 & 0.0385 & 0.4214 & 0.0104 & $\mathbf{0 . 3 9 6 9}$ & $\mathbf{0 . 0 0 8 1}$ \\
181079 & 0.4793 & 0.0424 & 0.4726 & 0.0491 & 0.4181 & 0.0172 & $\mathbf{0 . 3 9 6 6}$ & $\mathbf{0 . 0 1 3 5}$ \\
198054 & 0.5745 & 0.0625 & 0.5784 & 0.0433 & 0.4065 & 0.0101 & $\mathbf{0 . 3 9 3 3}$ & $\mathbf{0 . 0 0 1 7}$ \\
385028 & 0.4570 & 0.0599 & 0.4280 & 0.0747 & 0.3426 & $\mathbf{0 . 0 0 2 7}$ & $\mathbf{0 . 3 3 7 3}$ & 0.0029 \\
I1 & 0.1245 & 0.0048 & 0.1105 & 0.0286 & 0.104 & 0.0180 & $\mathbf{0 . 0 6 8 7}$ & $\mathbf{0 . 0 0 4 5}$ \\
I2 & 0.1661 & 0.0028 & 0.1696 & 0.0065 & 0.1103 & 0.0204 & $\mathbf{0 . 0 6 5 4}$ & $\mathbf{0 . 0 0 1 5}$ \\
I3 & 0.0602 & 0.0134 & 0.692 & 0.0456 & 0.1098 & 0.0370 & $\mathbf{0 . 0 4 3 3}$ & $\mathbf{0 . 0 3 4 4}$ \\
I4 & 0.3830 & 0.0287 & 0.3895 & 0.0261 & 0.3743 & $\mathbf{0 . 0 1 6 2}$ & $\mathbf{0 . 3 4 0 6}$ & 0.0252 \\
I5 & 0.2950 & 0.0518 & 0.2964 & 0.0632 & 0.2861 & $\mathbf{0 . 0 2 0 7}$ & $\mathbf{0 . 2 3 2 8}$ & 0.0218 \\
I6 & 0.3618 & 0.0292 & 0.4207 & 0.0199 & 0.3440 & $\mathbf{0 . 0 1 9 6}$ & $\mathbf{0 . 3 2 5 7}$ & 0.0275 \\
\hline
\end{tabular}

Table 9: Results of Wilcoxon signed rank test between HMS-based and conventional clustering-based algorithms

\begin{tabular}{lc}
\hline comparison & $p$-value \\
\hline ACHMS vs. $k$-means & $7.1263 \mathrm{E}-04$ \\
ACHMS vs. FCM & $2.9305 \mathrm{E}-04$ \\
\hline ACLSHMS vs. $k$-means & $2.9305 \mathrm{E}-04$ \\
ACLSHMS vs. FCM & $2.9305 \mathrm{E}-04$ \\
\hline
\end{tabular}

- Levine and Nazif's interclass contrast (LNIC) [45]: an unsupervised criterion based on the sum of contrasts of regions weighted by their areas. A higher LNIC value indicates better performance;

- Levine and Nazif's intra-class uniformity (LNIU) [45]: an unsupervised criterion that calculates the sum of normalised standard deviations of each region. A higher LNIU value indicates better performance;

- Variation of information (VOI) [46]: a supervised criterion that calculates the distance between two clusters in terms of the average conditional entropy of one cluster given the other. A lower VOI value indicates better performance;

- Probabilistic rand index (PRI) [47]: is the ratio of pixels whose labelling are consistent between the computed segmentation and the ground truth. A higher PRI value indicates better performance.

Table 10 gives the results for the BOR criterion in terms of the mean over the 25 runs and a ranking analysis. As we can note, our ACLSHMS algorithm performs best in 13 of the 17 cases and ranks 2-nd and 3-rd for two more images each. Overall, ACLSHMS clearly yields the best average rank and thus outperforms all other algorithms.

Table 11 lists the obtained results for the LNIC criterion. For 11 of the 17 images, ACLSHMS yields the best results, while ACHMS outperforms the other algorithms for 2 further images. Overall, ACLSHMS is again clearly the top-ranked algorithm.

Results for LNIU are shown in Table 12 and are broadly similar to the LNIC results, thus again demonstrating that our proposed ACLSHMS algorithm yields overall superior performance compared to all other algorithms. 
Table 10: BOR results for all images and algorithms.

\begin{tabular}{|c|c|c|c|c|c|c|c|c|}
\hline image & & ACGA & $\mathrm{ACDE}$ & ACPSO & ACHS & ACABC & ACHMS & ACLSHMS \\
\hline \multirow[t]{2}{*}{ Lenna } & mean & 0.469 & 0.0424 & 0.0509 & 0.0448 & 0.0468 & 0.0452 & 0.0438 \\
\hline & rank & 7 & 1 & 6 & 3 & 5 & 4 & 2 \\
\hline \multirow[t]{2}{*}{ Airplane } & mean & 0.491 & 0.0474 & 0.0464 & 0.0374 & 0.0374 & 0.0372 & 0.0355 \\
\hline & rank & 7 & 6 & 5 & 3.5 & 3.5 & 2 & 1 \\
\hline \multirow[t]{2}{*}{ House } & mean & 0.325 & 0.0319 & 0.0267 & 0.0246 & 0.0314 & 0.0230 & 0.0220 \\
\hline & rank & 7 & 6 & 4 & 3 & 5 & 2 & 1 \\
\hline \multirow[t]{2}{*}{ Peppers } & mean & 0.598 & 0.0591 & 0.0593 & 0.0572 & 0.0591 & 0.0590 & 0.0568 \\
\hline & rank & 7 & 4.5 & 6 & 2 & 4.5 & 3 & 1 \\
\hline \multirow[t]{2}{*}{ MRI } & mean & 0.315 & 0.0308 & 0.0309 & 0.0257 & 0.0250 & 0.0243 & 0.0242 \\
\hline & rank & 7 & 5 & 6 & 4 & 3 & 2 & 1 \\
\hline \multirow[t]{2}{*}{ Caspian Sea } & mean & 0.477 & 0.0466 & 0.0450 & 0.0450 & 0.0440 & 0.0385 & 0.0375 \\
\hline & rank & 7 & 6 & 4.5 & 4.5 & 3 & 2 & 1 \\
\hline \multirow[t]{2}{*}{198054} & mean & 0.463 & 0.0424 & 0.0349 & 0.0459 & 0.0429 & 0.0396 & 0.0278 \\
\hline & rank & 7 & 4 & 2 & 6 & 5 & 3 & 1 \\
\hline \multirow[t]{2}{*}{181079} & mean & 0.811 & 0.0760 & 0.0781 & 0.0717 & 0.0825 & 0.0767 & 0.0667 \\
\hline & rank & 7 & 3 & 5 & 2 & 6 & 4 & 1 \\
\hline \multirow[t]{2}{*}{385028} & mean & 0.572 & 0.0505 & 0.0501 & 0.0506 & 0.0551 & 0.0474 & 0.0458 \\
\hline & rank & 7 & 4 & 3 & 5 & 6 & 2 & 1 \\
\hline \multirow[t]{2}{*}{12003} & mean & 0.688 & 0.0645 & 0.0607 & 0.0554 & 0.0658 & 0.0691 & 0.0623 \\
\hline & rank & 7 & 4 & 2 & 1 & 5 & 6 & 3 \\
\hline \multirow[t]{2}{*}{42049} & mean & 0.588 & 0.0424 & 0.0429 & 0.0510 & 0.0567 & 0.0414 & 0.0332 \\
\hline & rank & 7 & 3 & 4 & 5 & 6 & 2 & 1 \\
\hline \multirow[t]{2}{*}{ I1 } & mean & $5.6232 \mathrm{E}-03$ & $3.9303 \mathrm{E}-04$ & 6.5999E-05 & $8.5111 \mathrm{E}-04$ & $3.9424 \mathrm{E}-04$ & $3.6518 \mathrm{E}-04$ & $1.3137 \mathrm{E}-04$ \\
\hline & rank & 7 & 4 & 1 & 6 & 5 & 3 & 2 \\
\hline \multirow[t]{2}{*}{$\mathrm{I} 2$} & mean & $1.0010 \mathrm{E}-03$ & 5.9492E-04 & $6.1208 \mathrm{E}-04$ & $1.3394 \mathrm{E}-03$ & $6.0918 \mathrm{E}-04$ & $5.9217 \mathrm{E}-04$ & $5.8868 \mathrm{E}-04$ \\
\hline & rank & 6 & 3 & 5 & 7 & 4 & 2 & 1 \\
\hline \multirow[t]{2}{*}{ I3 } & mean & $2.3652 \mathrm{E}-03$ & $3.4362 \mathrm{E}-06$ & $1.4067 \mathrm{E}-05$ & 5.3779E-06 & $7.2345 \mathrm{E}-06$ & $4.8531 \mathrm{E}-06$ & $1.0302 \mathrm{E}-06$ \\
\hline & rank & 7 & 2 & 6 & 4 & 5 & 3 & 1 \\
\hline \multirow[t]{2}{*}{ I4 } & mean & 0.0591 & 0.0385 & 0.0417 & 0.0559 & 0.0675 & 0.0334 & 0.0282 \\
\hline & rank & 6 & 3 & 4 & 5 & 7 & 2 & 1 \\
\hline \multirow[t]{2}{*}{ I5 } & mean & 0.0301 & 0.0192 & 0.0179 & 0.0212 & 0.0299 & 0.0150 & 0.0088 \\
\hline & rank & 7 & 4 & 3 & 5 & 6 & 2 & 1 \\
\hline \multirow[t]{2}{*}{ I6 } & mean & 0.0723 & 0.0345 & 0.0402 & 0.0461 & 0.0716 & 0.0268 & 0.0350 \\
\hline & rank & 7 & 2 & 4 & 5 & 6 & 1 & 3 \\
\hline average rank & & 6.88 & 3.79 & 4.15 & 4.18 & 5.00 & 2.65 & 1.35 \\
\hline overall rank & & 7 & 3 & 4 & 5 & 6 & 2 & 1 \\
\hline
\end{tabular}

The two supervised measures we employ, VOI and PRI, require a ground truth and thus we can only apply them on the images of the Berkeley segmentation dataset and the synthetic images. Table 13 presents the results for the VOI criterion. ACLSHMS is ranked first or second for all images which leads to the best average rank, followed by ACHMS, and thus a further indication that our algorithm outperforms other methods.

The PRI results are given in Table 14 . Here, once again ACLSHMS achieves the best overall rank to outperform all other algorithms.

Overall, our extensive evaluation clearly demonstrates that ACLSHMS represents a very powerful algorithm for image clustering that is shown to be superior to all other evaluated methods. The basis of this is the HMS algorithm which comprises powerful operators that lead to excellent performance. Consequently, ACHMS is shown to outperform the other metaheuristic algorithms based on all evaluated measures. The effectiveness of the employed local search strategy can be seen by comparing ACLSHMS with ACHMS. In the virtually all cases, ACLSHMS is shown to outperform ACHMS, confirming that the local search does indeed lead to improved intensification. As a result, ACLSHMS is demonstrated 
Table 11: LNIC results for all images and algorithms.

\begin{tabular}{|c|c|c|c|c|c|c|c|c|}
\hline image & & ACGA & ACDE & ACPSO & ACHS & ACABC & ACHMS & ACLSHMS \\
\hline \multirow[t]{2}{*}{ Lenna } & mean & 0.2263 & 0.3295 & 0.2241 & 0.3339 & 0.3455 & 0.3534 & 0.3614 \\
\hline & rank & 6 & 5 & 7 & 4 & 3 & 2 & 1 \\
\hline \multirow[t]{2}{*}{ Airplane } & mean & 0.1562 & 0.3061 & 0.1385 & 0.3042 & 0.3005 & 0.3073 & 0.3093 \\
\hline & rank & 6 & 3 & 7 & 4 & 5 & 2 & 1 \\
\hline \multirow[t]{2}{*}{ House } & mean & 0.2163 & 0.2282 & 0.1773 & 0.2319 & 0.2379 & 0.2356 & 0.2398 \\
\hline & rank & 6 & 5 & 7 & 4 & 2 & 3 & 1 \\
\hline \multirow[t]{2}{*}{ Peppers } & mean & 0.2512 & 0.3423 & 0.2589 & 0.3158 & 0.3307 & 0.3762 & 0.3797 \\
\hline & rank & 7 & 3 & 6 & 5 & 4 & 2 & 1 \\
\hline \multirow[t]{2}{*}{ MRI } & mean & 0.5521 & 0.6971 & 0.5403 & 0.6442 & 0.7244 & 0.7314 & 0.7408 \\
\hline & rank & 6 & 4 & 7 & 5 & 3 & 2 & 1 \\
\hline \multirow[t]{2}{*}{ Caspian Sea } & mean & 0.2163 & 0.2439 & 0.2146 & 0.2440 & 0.2419 & 0.2462 & 0.2477 \\
\hline & rank & 6 & 4 & 7 & 3 & 5 & 2 & 1 \\
\hline \multirow[t]{2}{*}{198054} & mean & 0.2563 & 0.4763 & 0.2547 & 0.4439 & 0.4734 & 0.4559 & 0.4561 \\
\hline & rank & 6 & 1 & 7 & 5 & 2 & 4 & 3 \\
\hline \multirow[t]{2}{*}{181079} & mean & 0.2361 & 0.3393 & 0.2194 & 0.3778 & 0.3843 & 0.3748 & 0.3796 \\
\hline & rank & 6 & 5 & 7 & 3 & 1 & 4 & 2 \\
\hline \multirow{2}{*}{385028} & mean & 0.2010 & 0.2492 & 0.1942 & 0.2734 & 0.2786 & 0.2764 & 0.2753 \\
\hline & rank & 6 & 5 & 7 & 4 & 1 & 2 & 3 \\
\hline \multirow[t]{2}{*}{12003} & mean & 0.4102 & 0.5031 & 0.4005 & 0.4788 & 0.5049 & 0.5144 & 0.5155 \\
\hline & rank & 6 & 4 & 7 & 5 & 3 & 2 & 1 \\
\hline \multirow[t]{2}{*}{42049} & mean & 0.1710 & 0.2043 & 0.1742 & 0.2123 & 0.2177 & 0.2212 & 0.2297 \\
\hline & rank & 7 & 5 & 6 & 4 & 3 & 2 & 1 \\
\hline \multirow[t]{2}{*}{ I1 } & mean & 0.1596 & 0.1873 & 0.1621 & 0.1869 & 0.1933 & 0.1845 & 0.1976 \\
\hline & rank & 7 & 3 & 6 & 4 & 2 & 5 & 1 \\
\hline \multirow[t]{2}{*}{$\mathrm{I} 2$} & mean & 0.2013 & 0.2228 & 0.2001 & 0.2203 & 0.2217 & 0.2226 & 0.2229 \\
\hline & rank & 6 & 2 & 7 & 5 & 4 & 3 & 1 \\
\hline \multirow[t]{2}{*}{ I3 } & mean & 0.4103 & 0.4196 & 0.4099 & 0.4034 & 0.4345 & 0.4489 & 0.4309 \\
\hline & rank & 5 & 4 & 6 & 7 & 2 & 1 & 3 \\
\hline \multirow[t]{2}{*}{ I4 } & mean & 0.2195 & 0.2815 & 0.2609 & 0.2275 & 0.2241 & 0.2625 & 0.2386 \\
\hline & rank & 7 & 1 & 3 & 5 & 6 & 2 & 4 \\
\hline \multirow[t]{2}{*}{ I5 } & mean & 0.2130 & 0.2140 & 0.2118 & 0.2147 & 0.2279 & 0.2436 & 0.2284 \\
\hline & rank & 6 & 5 & 7 & 4 & 3 & 1 & 2 \\
\hline \multirow[t]{2}{*}{ I6 } & mean & 0.3011 & 0.3035 & 0.3090 & 0.3057 & 0.3031 & 0.3159 & 0.3238 \\
\hline & rank & 7 & 5 & 3 & 4 & 6 & 2 & 1 \\
\hline average rank & & 6.24 & 3.76 & 6.29 & 4.41 & 3.24 & 2.41 & 1.65 \\
\hline overall rank & & 6 & 4 & 7 & 5 & 3 & 2 & 1 \\
\hline
\end{tabular}

to provide superior automatic clustering performance compared to all other evaluated methods based on all evaluation measure including a statistical analysis and a robustness analysis while also showing faster convergence.

\section{Conclusions}

Clustering-based image segmentation methods attempt to find pixel groups using criteria such as pixel intensity, so that pixels in the same group share similar features, while pixels from different groups should differ. In the past, algorithms like $k$-means have been popularly employed for this purpose but they suffer from drawbacks such as getting trapped in local optima and sensitivity to initialisation.

In this paper, we have proposed a novel automatic image clustering algorithm based on the human mental search (HMS) optimisation algorithm. Our ACLSHMS algorithm is able to automatically identify an optimal number of clusters simultaneously with the cluster the cluster configuration, while a local search mechanism is embedded to yield further 
Table 12: LNIU results for all images and algorithms.

\begin{tabular}{|c|c|c|c|c|c|c|c|c|}
\hline image & & ACGA & ACDE & ACPSO & ACHS & ACABC & ACHMS & ACLSHMS \\
\hline \multirow[t]{2}{*}{ Lenna } & mean & 0.0402 & 0.0507 & 0.0306 & 0.0523 & 0.0515 & 0.0568 & 0.0523 \\
\hline & rank & 6 & 5 & 7 & 2.5 & 4 & 1 & 2.5 \\
\hline \multirow[t]{2}{*}{ Airplane } & mean & 0.0512 & 0.0519 & 0.0322 & 0.0526 & 0.0530 & 0.0535 & 0.0555 \\
\hline & rank & 6 & 5 & 7 & 4 & 3 & 2 & 1 \\
\hline \multirow[t]{2}{*}{ House } & mean & 0.0251 & 0.0278 & 0.0242 & 0.0296 & 0.0224 & 0.0281 & 0.0299 \\
\hline & rank & 5 & 4 & 6 & 2 & 7 & 3 & 1 \\
\hline \multirow[t]{2}{*}{ Peppers } & mean & 0.0403 & 0.0587 & 0.0402 & 0.0552 & 0.0587 & 0.0628 & 0.0638 \\
\hline & rank & 6 & 3.5 & 7 & 5 & 3.5 & 2 & 1 \\
\hline \multirow[t]{2}{*}{ MRI } & mean & 0.0352 & 0.0507 & 0.0261 & 0.0500 & 0.0507 & 0.0559 & 0.0589 \\
\hline & rank & 6 & 3.5 & 7 & 5 & 3.5 & 2 & 1 \\
\hline \multirow[t]{2}{*}{ Caspian Sea } & mean & 0.0421 & 0.0474 & 0.0376 & 0.0422 & 0.0422 & 0.0482 & 0.0488 \\
\hline & rank & 6 & 3 & 7 & 4.5 & 4.5 & 2 & 1 \\
\hline \multirow[t]{2}{*}{198054} & mean & 0.0421 & 0.0555 & 0.0329 & 0.0584 & 0.0579 & 0.0643 & 0.0582 \\
\hline & rank & 6 & 5 & 7 & 2 & 4 & 1 & 3 \\
\hline \multirow[t]{2}{*}{181079} & mean & 0.0692 & 0.0773 & 0.0433 & 0.0733 & 0.0753 & 0.0757 & 0.0794 \\
\hline & rank & 6 & 2 & 7 & 5 & 4 & 3 & 1 \\
\hline \multirow{2}{*}{385028} & mean & 0.0621 & 0.0557 & 0.0365 & 0.0706 & 0.0706 & 0.0642 & 0.0706 \\
\hline & rank & 5 & 6 & 7 & 2 & 2 & 4 & 2 \\
\hline \multirow[t]{2}{*}{12003} & mean & 0.0452 & 0.0762 & 0.0486 & 0.0785 & 0.0821 & 0.0903 & 0.0857 \\
\hline & rank & 7 & 5 & 6 & 4 & 3 & 1 & 2 \\
\hline \multirow[t]{2}{*}{42049} & mean & 0.0429 & 0.0489 & 0.0386 & 0.0635 & 0.0661 & 0.0658 & 0.0694 \\
\hline & rank & 6 & 5 & 7 & 4 & 2 & 3 & 1 \\
\hline \multirow[t]{2}{*}{ I1 } & mean & 0.0010 & 0.0010 & 0.0005 & 0.0010 & 0.0010 & 0.0010 & 0.0011 \\
\hline & rank & 4 & 4 & 7 & 4 & 4 & 4 & 1 \\
\hline \multirow[t]{2}{*}{ I2 } & mean & 0.0012 & 0.0012 & 0.0012 & 0.0014 & 0.0016 & 0.0013 & 0.0014 \\
\hline & rank & 6 & 6 & 6 & 2.5 & 1 & 4 & 2.5 \\
\hline \multirow[t]{2}{*}{$\mathrm{I} 3$} & mean & $2.6358 \mathrm{E}-06$ & $3.1297 \mathrm{E}-06$ & $1.2619 \mathrm{E}-06$ & $5.6634 \mathrm{E}-06$ & $6.0668 \mathrm{E}-06$ & $3.8805 \mathrm{E}-06$ & $3.3485 \mathrm{E}-06$ \\
\hline & rank & 6 & 5 & 7 & 2 & 1 & 3 & 4 \\
\hline \multirow[t]{2}{*}{ I4 } & mean & 0.0390 & 0.0367 & 0.0394 & 0.0395 & 0.0312 & 0.0445 & 0.0447 \\
\hline & rank & 5 & 6 & 4 & 3 & 7 & 2 & 1 \\
\hline \multirow[t]{2}{*}{ I5 } & mean & 0.0121 & 0.0126 & 0.0105 & 0.0160 & 0.0136 & 0.0179 & 0.0149 \\
\hline & rank & 6 & 5 & 7 & 2 & 4 & 1 & 3 \\
\hline \multirow[t]{2}{*}{ I6 } & mean & 0.0310 & 0.0336 & 0.0347 & 0.0301 & 0.0376 & 0.0374 & 0.0417 \\
\hline & rank & 6 & 5 & 4 & 7 & 2 & 3 & 1 \\
\hline average rank & & 5.76 & 4.59 & 6.47 & 3.56 & 3.50 & 2.41 & 1.71 \\
\hline overall rank & & 6 & 5 & 7 & 4 & 3 & 2 & 1 \\
\hline
\end{tabular}

improved efficacy.

To evaluate the performance of our proposed approach, we have conducted an extensive set of experiments in which we have also compared ACLSHMS with existing state-of-the-art algorithms. We have shown that, on a benchmark set of challenging images, our algorithm gives the best results in terms of the commonly employed DB index and also that this superior performance is statistically significant, while the algorithm is also confirmed to be more robust than other approaches. Equally, we have demonstrated that the local search mechanism does indeed lead to improved performance and that ACLSHMS is better at estimating the true number of clusters compared to other methods. Further, and importantly, we have evaluated the image segmentation performance using both supervised and unsupervised criteria to show that ACLSHMS also offers superior efficacy in this respect.

In this paper, we have used the pixel intensity as the feature employed for clustering and thus in the conducted experiments we have utilised greyscale images. Our proposed method can however be easily extended to colour or other multi-channel representations, while other cost functions can also be considered. 
Table 13: VOI results for Berkeley images and all algorithms.

\begin{tabular}{|c|c|c|c|c|c|c|c|c|}
\hline \multicolumn{2}{|l|}{ image } & ACGA & ACDE & ACPSO & ACHS & ACABC & ACHMS & ACLSHMS \\
\hline \multirow[t]{2}{*}{198054} & mean & 2.3765 & 2.3647 & 2.8795 & 2.3694 & 2.3638 & 2.3445 & 2.2450 \\
\hline & rank & 6 & 4 & 7 & 5 & 3 & 2 & 1 \\
\hline \multirow[t]{2}{*}{181079} & mean & 3.3940 & 3.1828 & 4.0570 & 3.6325 & 3.1576 & 2.9794 & 3.1148 \\
\hline & rank & 5 & 4 & 7 & 6 & 3 & 1 & 2 \\
\hline \multirow[t]{2}{*}{385028} & mean & 3.3663 & 3.1607 & 3.6942 & 2.9692 & 3.1533 & 3.0224 & 3.0034 \\
\hline & rank & 6 & 5 & 7 & 1 & 4 & 3 & 2 \\
\hline \multirow[t]{2}{*}{12003} & mean & 2.2544 & 2.0401 & 2.5744 & 2.1271 & 2.0259 & 1.9915 & 2.0217 \\
\hline & rank & 6 & 4 & 7 & 5 & 3 & 1 & 2 \\
\hline \multirow[t]{2}{*}{42049} & mean & 4.4215 & 3.7786 & 4.1039 & 3.6922 & 3.5437 & 3.6834 & 3.6233 \\
\hline & rank & 7 & 5 & 6 & 4 & 1 & 3 & 2 \\
\hline \multirow[t]{2}{*}{ I1 } & mean & 1.6541 & 0.2358 & 0.2581 & 0.2476 & 0.2353 & 0.2329 & 0.2179 \\
\hline & rank & 7 & 4 & 6 & 5 & 3 & 2 & 1 \\
\hline \multirow[t]{2}{*}{$\mathrm{I} 2$} & mean & 1.8923 & 0.3797 & 0.3897 & 0.3957 & 0.3797 & 0.3797 & 0.3796 \\
\hline & rank & 7 & 3 & 5 & 6 & 3 & 3 & 1 \\
\hline \multirow[t]{2}{*}{ I3 } & mean & 3.3155 & 2.3412 & 2.4404 & 2.3052 & 2.3783 & 2.2114 & 2.2903 \\
\hline & rank & 7 & 4 & 6 & 3 & 5 & 1 & 2 \\
\hline \multirow[t]{2}{*}{ I4 } & mean & 2.4531 & 2.4437 & 2.3860 & 2.1335 & 2.1887 & 2.2931 & 2.1853 \\
\hline & rank & 7 & 6 & 5 & 1 & 3 & 4 & 2 \\
\hline \multirow[t]{2}{*}{ I5 } & mean & 3.8330 & 1.5622 & 1.5388 & 1.6083 & 1.7500 & 1.4515 & 1.3031 \\
\hline & rank & 7 & 4 & 3 & 5 & 6 & 2 & 1 \\
\hline \multirow[t]{2}{*}{ I6 } & mean & 4.2728 & 1.3503 & 1.5892 & 1.6801 & 2.1983 & 1.2199 & 1.2558 \\
\hline & rank & 7 & 3 & 4 & 5 & 6 & 1 & 2 \\
\hline \multicolumn{2}{|c|}{ average rank } & 6.45 & 4.18 & 5.72 & 4.18 & 3.64 & 2.09 & 1.64 \\
\hline \multicolumn{2}{|c|}{ overall rank } & 7 & 4.5 & 6 & 4.5 & 3 & 2 & 1 \\
\hline
\end{tabular}

Table 14: PRI results for Berkeley images and all algorithms.

\begin{tabular}{|c|c|c|c|c|c|c|c|c|}
\hline \multicolumn{2}{|l|}{ image } & ACGA & ACDE & ACPSO & ACHS & $\mathrm{ACABC}$ & ACHMS & ACLSHMS \\
\hline \multirow[t]{2}{*}{198054} & mean & 0.5234 & 0.5375 & 0.5551 & 0.5294 & 0.5395 & 0.5476 & 0.5897 \\
\hline & rank & 7 & 5 & 2 & 6 & 4 & 3 & 1 \\
\hline \multirow[t]{2}{*}{181079} & mean & 0.3166 & 0.5705 & 0.5527 & 0.5731 & 0.5589 & 0.5905 & 0.5872 \\
\hline & rank & 7 & 4 & 6 & 3 & 5 & 1 & 2 \\
\hline \multirow[t]{2}{*}{385028} & mean & 0.3332 & 0.6375 & 0.6175 & 0.6277 & 0.6205 & 0.6486 & 0.6496 \\
\hline & rank & 7 & 3 & 6 & 4 & 5 & 2 & 1 \\
\hline \multirow[t]{2}{*}{12003} & mean & 0.3186 & 0.7447 & 0.7355 & 0.7412 & 0.7375 & 0.7469 & 0.7437 \\
\hline & rank & 7 & 2 & 6 & 4 & 5 & 1 & 3 \\
\hline \multirow[t]{2}{*}{42049} & mean & 0.2817 & 0.6985 & 0.6958 & 0.6426 & 0.6208 & 0.6715 & 0.7021 \\
\hline & rank & 7 & 2 & 3 & 5 & 6 & 4 & 1 \\
\hline \multirow[t]{2}{*}{ I1 } & mean & 0.3854 & 0.9817 & 0.9832 & 0.9766 & 0.9817 & 0.9819 & 0.9827 \\
\hline & rank & 7 & 5.5 & 1 & 5.5 & 4 & 3 & 2 \\
\hline \multirow[t]{2}{*}{$\mathrm{I} 2$} & mean & 0.3821 & 0.9753 & 0.9668 & 0.9701 & 0.9753 & 0.9753 & 0.9753 \\
\hline & rank & 7 & 2.5 & 6 & 5 & 2.5 & 2.5 & 2.5 \\
\hline \multirow[t]{2}{*}{ I3 } & mean & 0.1793 & 0.2160 & 0.2137 & 0.2153 & 0.2201 & 0.2246 & 0.2246 \\
\hline & rank & 7 & 4 & 6 & 5 & 3 & 1.5 & 1.5 \\
\hline \multirow[t]{2}{*}{ I4 } & mean & 0.4292 & 0.6763 & 0.6598 & 0.6754 & 0.6736 & 0.6625 & 0.6799 \\
\hline & rank & 7 & 2 & 6 & 3 & 4 & 5 & 1 \\
\hline \multirow[t]{2}{*}{ I5 } & mean & 0.2435 & 0.9197 & 0.9191 & 0.9147 & 0.8926 & 0.9304 & 0.9476 \\
\hline & rank & 7 & 3 & 4 & 5 & 6 & 2 & 1 \\
\hline \multirow[t]{2}{*}{ I6 } & mean & 0.4821 & 0.9063 & 0.8803 & 0.8651 & 0.7941 & 0.9126 & 0.9126 \\
\hline & rank & 7 & 3 & 4 & 5 & 6 & 1.5 & 1.5 \\
\hline \multicolumn{2}{|c|}{ average rank } & 7.00 & 3.28 & 4.55 & 4.59 & 4.59 & 2.40 & 1.59 \\
\hline \multicolumn{2}{|c|}{ overall rank } & 7 & 3 & 4 & 5.5 & 5.5 & 2 & 1 \\
\hline
\end{tabular}

\section{References}

[1] S. Das and A. Konar, "Automatic image pixel clustering with an improved differential evolution," Applied Soft Computing, vol. 9, no. 1, pp. 226-236, 2009. 
[2] S. H. R. Sanei and R. S. Fertig III, "Uncorrelated volume element for stochastic modeling of microstructures based on local fiber volume fraction variation," Composites Science and Technology, vol. 117, pp. 191-198, 2015.

[3] S. H. R. Sanei, E. J. Barsotti, D. Leonhardt, and R. S. Fertig III, "Characterization, synthetic generation, and statistical equivalence of composite microstructures," Journal of Composite Materials, vol. 51, no. 13, pp. 1817-1829, 2017.

[4] U. Jamil, A. Sajid, M. Hussain, O. Aldabbas, A. Alam, and M. U. Shafiq, "Melanoma segmentation using biomedical image analysis for smarter mobile healthcare," Journal of Ambient Intelligence and Humanized Computing, pp. 1-22, 2019.

[5] R. A. Rose and A. Annadhason, "GHT based automatic kidney image segmentation using modified AAM and GBDT," Health and Technology, pp. 1-10, 2019.

[6] S. J. Mousavirad, H. Ebrahimpour-Komleh, and G. Schaefer, "Effective image clustering based on human mental search," Applied Soft Computing, vol. 78, pp. 209-220, 2019.

[7] J. MacQueen, "Some methods for classification and analysis of multivariate observations," in 5th Berkeley Symposium on Mathematical Statistics and Probability, pp. 281-297, Oakland, CA, USA, 1967.

[8] R. L. Cannon, J. V. Dave, and J. C. Bezdek, "Efficient implementation of the fuzzy c-means clustering algorithms," IEEE Transactions on Pattern Analysis and Machine Intelligence, vol. 8, no. 2, pp. 248-255, 1986.

[9] E.-G. Talbi, Metaheuristics: from design to implementation. John Wiley \& Sons, 2009.

[10] R. Storn and K. Price, "Differential evolution-a simple and efficient heuristic for global optimization over continuous spaces," Journal of Global Optimization, vol. 11, no. 4, pp. 341-359, 1997.

[11] Y. Shi and R. Eberhart, "A modified particle swarm optimizer," in IEEE International Conference on Evolutionary Computation, pp. 69-73, 1998.

[12] Z. W. Geem, J. H. Kim, and G. V. Loganathan, “A new heuristic optimization algorithm: harmony search,” Simulation, vol. 76, no. 2, pp. 60-68, 2001.

[13] A. S. Fraser, "Simulation of genetic systems by automatic digital computers ii. effects of linkage on rates of advance under selection," Australian Journal of Biological Sciences, vol. 10, no. 4, pp. 492-500, 1957.

[14] H. J. Bremermann, The evolution of intelligence: The nervous system as a model of its environment. University of Washington, Department of Mathematics, 1958.

[15] J. H. Holland, Adaptation in natural and artificial systems. MIT Press, 1992.

[16] P. Scheunders, “A genetic Lloyd-Max image quantization algorithm,” Pattern Recognition Letters, vol. 17, no. 5, pp. 547-556, 1996. 
[17] M. Awad, K. Chehdi, and A. Nasri, "Multi-component image segmentation using a hybrid dynamic genetic algorithm and fuzzy c-means," IET Image Processing, vol. 3, no. 2, pp. 52-62, 2009.

[18] M. G. Omran, A. P. Engelbrecht, and A. Salman, "Image classification using particle swarm optimization," in Recent Advances in Simulated Evolution and Learning, pp. 347-365, World Scientific, 2004.

[19] J. Yu, "A novel chaos PSO clustering algorithm for texture image segmentation," in Recent Advances in Computer Science and Information Engineering, pp. 269-274, Springer, 2012.

[20] M. Omran and S. Al-Sharhan, "Barebones particle swarm methods for unsupervised image classification," in IEEE Congress on Evolutionary Computation, pp. 3247-3252, 2007.

[21] N. Dhanachandra and Y. J. Chanu, "An image segmentation approach based on fuzzy c-means and dynamic particle swarm optimization algorithm," Multimedia Tools and Applications, pp. 1-20, 2020.

[22] W. Kwedlo, "A clustering method combining differential evolution with the $k$-means algorithm," Pattern Recognition Letters, vol. 32, no. 12, pp. 1613-1621, 2011.

[23] C. Ozturk, E. Hancer, and D. Karaboga, "Improved clustering criterion for image clustering with artificial bee colony algorithm," Pattern Analysis and Applications, vol. 18, no. 3, pp. 587-599, 2015.

[24] L. Wang, Y. Yufeng, and J. Liu, "Clustering with a novel global harmony search algorithm for image segmentation," International Journal of Hybrid Information Technology, vol. 9, no. 2, pp. 183-194, 2016.

[25] S. Das, A. Abraham, and A. Konar, "Automatic clustering using an improved differential evolution algorithm," IEEE Transactions on Systems, Man, and Cybernetics-Part A: Systems and Humans, vol. 38, no. 1, pp. 218-237, 2008.

[26] C. Ozturk, E. Hancer, and D. Karaboga, "Dynamic clustering with improved binary artificial bee colony algorithm," Applied Soft Computing, vol. 28, pp. 69-80, 2015.

[27] D. Wolpert and G. Macready, "No free lunch theorems for optimization," IEEE Transactions on Evolutionary Computation, vol. 1, no. 1, pp. 67-82, 1997.

[28] M. G. Omran, A. Salman, and A. P. Engelbrecht, "Dynamic clustering using particle swarm optimization with application in image segmentation," Pattern Analysis and Applications, vol. 8, no. 4, p. 332, 2006.

[29] V. Kumar, J. K. Chhabra, and D. Kumar, "Automatic data clustering using parameter adaptive harmony search algorithm and its application to image segmentation,” Journal of Intelligent Systems, vol. 25, no. 4, pp. 595-610, 2016.

[30] R. R. Kurada, K. K. Pavan, and A. A. Rao, "Automatic teaching-learning-based optimization: A novel clustering method for gene functional enrichments," in Computational Intelligence Techniques for Comparative Genomics, pp. 17-35, Springer, 2015. 
[31] T. Niknam, E. T. Fard, N. Pourjafarian, and A. Rousta, "An efficient hybrid algorithm based on modified imperialist competitive algorithm and K-means for data clustering," Engineering Applications of Artificial Intelligence, vol. 24, no. 2, pp. 306-317, 2011.

[32] J. Tvrdik and I. Křivỳ, "Hybrid differential evolution algorithm for optimal clustering," Applied Soft Computing, vol. 35, pp. 502-512, 2015.

[33] E. Mehdizadeh, M. Teimouri, A. Zaretalab, and S. Niaki, "A combined approach based on K-means and modified electromagnetism-like mechanism for data clustering," International Journal of Information Technology $\mathcal{E}$ Decision Making, vol. 16, no. 05, pp. 1279-1307, 2017.

[34] A. Hatamlou, S. Abdullah, and H. Nezamabadi-Pour, "A combined approach for clustering based on K-means and gravitational search algorithms," Swarm and Evolutionary Computation, vol. 6, pp. 47-52, 2012.

[35] S. J. Mousavirad and H. Ebrahimpour-Komleh, "Human mental search: a new population-based metaheuristic optimization algorithm," Applied Intelligence, vol. 47, no. 3, pp. 850-887, 2017.

[36] S. J. Mousavirad and H. Ebrahimpour-Komleh, "Human mental search-based multilevel thresholding for image segmentation," Applied Soft Computing, 2019.

[37] D. L. Davies and D. W. Bouldin, “A cluster separation measure," IEEE Transactions on Pattern Analysis and Machine Intelligence, vol. 1, no. 2, pp. 224-227, 1979.

[38] R. Liu, B. Zhu, R. Bian, Y. Ma, and L. Jiao, "Dynamic local search based immune automatic clustering algorithm and its applications," Applied Soft Computing, vol. 27, pp. 250-268, 2015.

[39] D. Martin, C. Fowlkes, D. Tal, and J. Malik, "A database of human segmented natural images and its application to evaluating segmentation algorithms and measuring ecological statistics," in 8th International Conference on Computer Vision, vol. 2, pp. 416-423, 2001.

[40] Y. Liu, X. Wu, and Y. Shen, “Automatic clustering using genetic algorithms," Applied Mathematics and Computation, vol. 218, no. 4, pp. 1267-1279, 2011.

[41] P. N. Suganthan, N. Hansen, J. J. Liang, K. Deb, Y.-P. Chen, A. Auger, and S. Tiwari, "Problem definitions and evaluation criteria for the CEC 2005 special session on real-parameter optimization,” tech. rep., Nanyang Technological University Singapore, 2005.

[42] D. Karaboga and B. Akay, "A comparative study of artificial bee colony algorithm," Applied Mathematics and Computation, vol. 214, no. 1, pp. 108-132, 2009.

[43] J. Derrac, S. García, D. Molina, and F. Herrera, "A practical tutorial on the use of nonparametric statistical tests as a methodology for comparing evolutionary and swarm intelligence algorithms," Swarm and Evolutionary Computation, vol. 1, no. 1, pp. 3-18, 2011. 
[44] M. Borsotti, P. Campadelli, and R. Schettini, "Quantitative evaluation of color image segmentation results," Pattern Recognition Letters, vol. 19, no. 8, pp. 741-747, 1998.

[45] M. D. Levine and A. M. Nazif, "Dynamic measurement of computer generated image segmentations," IEEE Transactions on Pattern Analysis and Machine Intelligence, vol. 7, no. 2, pp. 155-164, 1985.

[46] M. Meil, "Comparing clusterings: an axiomatic view," in 22nd International Conference on Machine Learning, pp. 577-584, 2005.

[47] C. Pantofaru and M. Hebert, “A comparison of image segmentation algorithms," tech. rep., Robotics Institute, 2005. 